\title{
A bound on the entropy of supergravity?
}

\author{
Jan de Boer, ${ }^{a}$ Sheer El-Showk, ${ }^{a}$ llies Messamah ${ }^{a}$ and Dieter Van den Bleeken ${ }^{b}$ \\ ${ }^{a}$ Instituut voor Theoretische Fysica, Universiteit Amsterdam, \\ Valckenierstraat 65, 1018XE Amsterdam, The Netherlands \\ ${ }^{b}$ NHETC and Department of Physics and Astronomy, Rutgers University, \\ Piscataway, NJ 08855, U.S.A.
}

\begin{abstract}
We determine, in two independent ways, the number of BPS quantum states arising from supergravity degrees of freedom in a system with fixed total D4D0 charge. First, we count states generated by quantizing the spacetime degrees of freedom of "entropyless" multicentered solutions consisting of $\overline{\mathrm{D} 0}$-branes bound to a D6 $\overline{\mathrm{D} 6}$ pair. Second, we determine the number of free supergravity excitations of the corresponding $\mathrm{AdS}_{3}$ geometry with the same total charge. We find that, although these two approaches yield a priori different sets of states, the leading degeneracies in a large charge expansion are equal to each other and that, furthermore, the number of such states is parametrically smaller than that arising from the D4D0 black hole's entropy. This strongly suggests that supergravity alone is not sufficient to capture all degrees of freedom of large supersymmetric black holes. Comparing the free supergravity calculation to that of the D6 $\overline{\mathrm{D} 6} \mathrm{D} 0$ system we find that the bound on the free spectrum imposed by the stringy exclusion principle (a unitarity bound in the dual CFT) seems to be captured in the dynamics of the fully interacting but classcial supergravity equations of motion.
\end{abstract}

KeYwords: Black Holes in String Theory, AdS-CFT Correspondence, D-branes

ARXiv EPRINT: 0906.0011 


\section{Contents}

1 Introduction 1

2 Counting dipole halo states $\quad 5$

2.1 The dipole halo solution space 5

2.1.1 Charges and constraints 5

$\begin{array}{lll}2.1 .2 & \text { Scaling solutions } & 7\end{array}$

$\begin{array}{lll}2.1 .3 & \text { Symplectic form } & 7\end{array}$

2.1.4 Physical picture 9

2.2 The dipole halo states 11

$\begin{array}{lll}2.2 .1 & \text { States and polytopes } & 12\end{array}$

$\begin{array}{ll}\text { 2.2.2 The D6 } \overline{\mathrm{D} 6} \mathrm{D} 0 \text { partition function } & 14\end{array}$

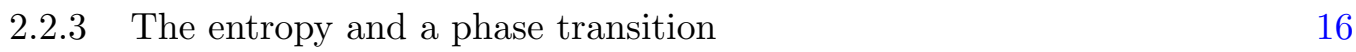

$\begin{array}{lll}2.3 & \text { Free supergravity estimate } & 18\end{array}$

$\begin{array}{lll}2.3 .1 & \text { Superconformal quantum numbers } & 19\end{array}$

2.3.2 The spectrum of BPS states 20

2.3.3 Comparison to black hole entropy and the stringy exclusion principle 21

3 Physical properties of scaling solutions $\quad 23$

3.1 Scaling solutions with more than 3 centers 24

3.2 Macroscopic quantum fluctuations from AdS/CFT 25

$\begin{array}{lll}3.3 & \text { Entropy of the } J=0 \text { locus } & 26\end{array}$

A The number of states as discrete points inside the polytope $\quad 27$

$\begin{array}{lll}\text { A.1 Evaluation of } \operatorname{det} \partial_{i} \partial_{j} g & 29\end{array}$

\section{Introduction}

The D4D0 BPS black hole was the first four dimensional black hole to enjoy a microscopic statistical interpretation of its entropy. In [1] it was shown that the five dimensional uplift of this black hole to a black string is dual to a $(0,4)$ CFT. If $q_{0}$ and $p^{A}$ denote the D0 and D4 charges of the black hole, then to leading order for large charges the central charge of the CFT equals $c=d_{A B C} p^{A} p^{B} p^{C}$, with $d_{A B C}$ the intersection numbers of the Calabi-Yau. We will abbreviate $d_{A B C} p^{A} p^{B} p^{C}$ by $p^{3}$ in what follows. If in addition $-q_{0} \gg p^{3} \gg 1$, Cardy's formula implies that the CFT has $e^{2 \pi \sqrt{\frac{-q_{0} p^{3}}{6}}}$ BPS states with the same quantum numbers as the black hole. This coincides exactly with the gravitational entropy, proportional to the black hole horizon, as given by the Bekenstein-Hawking formula. Although this determination of the number of black hole degrees of freedom using AdS/CFT duality is an amazing and beautiful result it is also partially unsatisfactory as it leaves a direct 
understanding of the black hole states in the strongly coupled or gravity regime unfulfilled. Motivated by this question we will consider a certain subset of BPS states that carry the same quantum numbers as the D4D0 black hole directly in their backreacted supergravity description.

The BPS states we consider arise from quantizing the supergravity solutions corresponding to D6 $\overline{\mathrm{D} 6} \mathrm{D} 0$ bound states, ${ }^{1}$ and can be studied using the techniques we developed in [2]. These solutions are horizon-(and hence entropy-)less ${ }^{2}$ multicentered configurations [3-6] that come in discrete sets of continuous families, parameterized by the possible equilibrium positions of the centers and the number and charges of the D0 centers. We refer to this continuous deformation space as a "solution space" and in [2] we showed that, due to the presence of intrinsic angular momentum coming from crossed electric and magnetic fields, this space is naturally interpreted as (a regular part of) the phase-space of the system and is thus amenable to geometric quantization. Using partition function techniques we calculate the number of states associated to all the possible solution spaces, classified by partitions of the total D0 charge, and find that, in the large charge regime, there is an exponential number, $d_{N, I}$, of D6 $\overline{\mathrm{D} 6} \mathrm{D} 0$ BPS states. The precise result we obtain in this paper depends on the value of the total D0 charge, $q_{0}$, relative to the D4 charge cubed $p^{3}$ :

$$
\log d_{N, I}= \begin{cases}\left(\alpha \frac{N^{2}}{4}\right)^{1 / 3} & \text { if } N \leq I \\ \left(\alpha \frac{I}{2}\left(N-\frac{I}{2}\right)\right)^{1 / 3} & \text { if } I \leq N\end{cases}
$$

with $I=\frac{p^{3}}{6}, N=-q_{0}+\frac{p^{3}}{24}$ and $\alpha=\frac{3}{4} \zeta(3)$.

This result reveals two interesting facts. First, by comparing to the number of states of the D4D0 black hole, given above, we see that for large charges the black hole entropy is exponentially larger. In this sense the BPS states we considered are extremely sparse in the set of states forming the black hole. Even though the set of states we obtain from supergravity is too small to dominate and thus capture the physics of large black holes, it does appear to have some rather intriguing physics of its own. As can be seen from the formula above there appears to be a phase transition at $N=I$ between two different regimes. It follows from our calculation that this is essentially due to a restriction on the orientation and size of the total angular momentum of the system, acting as a cutoff on the spectrum, leading to a smaller growth of states once $N>I$. More precisely, by the BPS equations of motion the total angular momentum is forced to always be positive, i.e. always pointing from $\overline{\mathrm{D} 6}$ to D6 along the axis through those centers, and to be smaller than $\frac{I}{2}$.

Faced with the result that the BPS states of the D6 $\overline{\mathrm{D} 6} \mathrm{D} 0$ supergravity solutions are insufficient to account for the D4D0 black hole entropy, one might wonder if there are other, perhaps more complicated, zero entropy BPS supergravity solutions that might generate

\footnotetext{
${ }^{1}$ As explained in the bulk of the paper the D6 and $\overline{\mathrm{D} 6}$ carry worldvolume flux leading to a total D4D0 charge for such a bound state.

${ }^{2}$ Note that there is a small degeneracy of $\chi\left(C Y_{3}\right)$ from the degrees of freedom of the D0 in the compactified dimensions. We neglect this fact in most parts of the paper, because it only changes the prefactor of the entropy rather than its scaling in the charges. This is discussed in more detail in section 2.3.
} 
enough states to account for some finite fraction of the black hole entropy. The second result of this paper is a computation indicating that this might not be the case. We compute the number of multiparticle linearized $\mathcal{N}=1$ (in 5-d) supergravity BPS modes around $\mathrm{AdS}_{3} \times \mathrm{S}^{2}$, the vacuum of the $(0,4) \mathrm{CFT}$ that contains the black hole ensemble. The result of this computation is an exponential number of states arising in the free theory, identical to what we found for the D6 $\overline{\mathrm{D} 6} \mathrm{D} 0$ system discussed above, including the phase transition (though the latter only emerges after imposing the stringy exclusion principle i.e. a unitarity constraint - on the free spectrum).

This might indicate that in the full, interacting theory the D6 $\overline{\mathrm{D} 6} \mathrm{D} 0$ states dominate the entropy obtainable from quantizing solutions of $5 \mathrm{~d} \mathcal{N}=1$ supergravity. There are, however, many subtleties that could spoil such a hasty conclusion. First of all, the D6 $\overline{\mathrm{D} 6} \mathrm{D} 0$ bound states are solutions of the fully interacting theory but when more closely analyzed the BPS constraint equations imply that they are composed primarily of non-interacting bits with only the $\mathrm{D} 6 \overline{\mathrm{D} 6}$ core providing a weak form of interactions between the centers. As pointed out in [7] these configurations can essentially be spectrally flowed to a gas of weakly interacting gravitons on an $\mathrm{AdS}_{3} \times \mathrm{S}^{2}$ background. Thus perhaps it is not so great a surprise that the degeneracy of such configurations is captured by the free theory.

We can, however, also compute the index in the free theory and one might expect this to fully agree with the degeneracy of the interacting theory. It is not clear, however, that the degrees of freedom captured by the index in the free theory do not undergo a phase transition when continued to nonzero coupling so there might still exist other, more intricate, supergravity solutions that do have a larger degeneracy than the D6 $\overline{\mathrm{D} 6} \mathrm{D} 0$ but that cease to exist in the free limit. We are not aware of a single solution that has this property however.

Another interesting observation that follows from the counting in the free theory is that there the cutoff on the spectrum is provided by the stringy exclusion principle [8], a unitarity bound following from the $(0,4)$ superconformal algebra, which must be enforced by hand. Comparing the free calculation and the D6 $\overline{\mathrm{D} 6} \mathrm{D} 0$ bound states we see that the bound given by this stringy exclusion principle seems to be encoded in the interacting theory through the BPS equations of motion which put constraints on the positions of the various centers of the D6 $\overline{\mathrm{D} 6} \mathrm{D} 0$ system such that the total angular momentum is bounded, $J_{3} \geq 0$. The latter bound on the angular momentum is equivalent to the stringy exclusion principle. This is somewhat surprising as it was previously suggested [8] that supergravity would not capture this bound. Although we have no explicit proof that the most general solution of supergravity satisfies this bound, the fact that it appears to be satisfied by a certain subset of solutions is certainly intriguing.

Finally we would like to discuss the relevance of our results to some of the many applications of these or similar multicentered solutions in the literature. There is a much larger class of similar solutions that in 5 dimensions correspond to entropy-less or smooth solutions $[5,6]$ and which are asymptotically indistinguishable from black holes/rings. Such geometries are often referred to (for better or for worse) as black hole "microstate" geometries as they are believed to play a roll in the fuzzball conjecture, see [9-13] for some reviews. As discussed in [14], even if the vast majority of black hole states arise 
from stringy excitations we may still hope to extract useful information from supergravity states if they are suitably dense in the black hole Hilbert space. Precisely what "suitably dense" means is not clear but at the very least one would imagine the entropy of all such configurations should scale in the same way as the total black hole entropy with the charges (though perhaps with a different coefficient). Our results are, at first sight, rather discouraging for the (supergravity based) microstate program. This because, at least in the class of solutions we consider, our results suggest that generic microstates will not be accessible within supergravity as these solutions have a parametrically smaller degeneracy than that required to capture the black hole's entropy. Thus to find a generic microstate and determine its spatial properties may require incorporating string or brane degrees of freedom which is technically quite hard, for an in depth discussion see [13]. We pointed out above that there remains the important disclaimer that we were not able to explicitly show that there are no other entropy-less supergravity configurations that might generate a large enough degeneracy, although we have shown that if such configurations exist they would have to disappear in the free limit around $\mathrm{AdS}_{3}$.

Another approach to the interpretation of black hole states $[15,16]$ is also related, as shown in [17], to the D6 $\overline{\mathrm{D} 6} \mathrm{D} 0$ system studied here. In $[17,18]$ arguments were given that, if in the scaling regime the D0-branes of such a D6 $\overline{\mathrm{D} 6} \mathrm{D} 0$ bound state blow up to D2-branes through a version of the Myers effect, the quantization of these solutions would reproduce the entropy of the corresponding D4D0 black hole through the same Landau degeneracies as found in [15]. Our results seem to further confirm that such non-Abelian degrees of freedom are essential to produce enough degeneracy as our analysis of the system of [17] does not incorporate such non-Abelian stringy effects and yields too few states. ${ }^{3}$ Where in [15] each D2 brane with given induced D0 charge occupied one of $\sim p^{3}$ landau levels in the Calabi-Yau we find that in our quantization the addition of a single D0 to the D6 $\overline{\mathrm{D} 6}$ systems seems to also yield a growth in the degeneracy of order $p^{3}$. The difference in our case is, however, that the number of states gets suppressed as $1 / n$ ! for $n$ D0 centers due to the bound on the angular momentum leading to our observed growth with a power of $1 / 3$ in the charges, rather than the power of $1 / 2$ that the black hole entropy exhibits and which was reproduced in [15]. As this is an effect that emerges only after taking into account the full back reaction of the D0's and dominates for $n \gg 1$ one might wonder if the approximation of [15], neglecting the backreaction of the D0's on the geometry, might have to be corrected in a non-trivial way.

The paper is organized as follows. In section 2 we compute the entropy of asymptotically $\mathrm{AdS}_{3}$ states, first by restricting to a large class of fully backreacted solutions and then in the free theory and we show that these match. We begin by recalling, in section 2.1, a convenient coordinatization of the $\mathrm{D} 6 \overline{\mathrm{D} 6} \mathrm{D} 0$ system and quickly review the quantization of this system following [2]. In section 2.2 we study the partition function of this system and use it to determine the degeneracy at large charges. A similar computation is done for the

\footnotetext{
${ }^{3}$ Of course, non-Abelian degrees of freedom are part of the open string description of the system, and since our goal is to find a closed string description of the degrees of freedom we should eventually avoid nonAbelian degrees of freedom. Such a description could e.g. be that of fully back-reacted spherical D2-branes, whose explicit form is presently unknown. A first approach to this problem is made in [19].
} 
partition function of supergravity linearized around a background global $\mathrm{AdS}_{3} \times \mathrm{S}^{2}$ metric in section 2.3. In the free theory the stringy exclusion principle must be introduced by hand and changes the growth of states at $N>I$ whereas this transition emerges naturally from the $\mathrm{D} 6 \overline{\mathrm{D} 6} \mathrm{D} 0$ partition function. In section 3 we turn our attention to some other questions of relevance for the physics of these solutions. We provide an argument, complementary to that of [2], based on AdS/CFT, for the existence of macroscopic quantum fluctuations in section 3.2. To address a long-standing debate regarding whether it is the components of angular momentum coming from pairs of centers or only the total angular momentum that is quantized we use some mathematical results to study the $J=0$ submanifold of a general phase space in section 3.3. We find that, roughly speaking, all angular momenta are quantized but that the respective quantum numbers are not all independent. Some technical results regarding counting states on our phase spaces regarding as polytopes of toric manifolds are derived in appendix A.

\section{Counting dipole halo states}

In [2] the solution space associated with a D6 $\overline{\mathrm{D} 6}$ pair (with intersection product $I$ given below) surrounded by $N$ D0's fixed in the plane orthogonal to the $\mathrm{D} 6 \overline{\mathrm{D} 6}$ axis, a system we will refer to as the dipole halo, was quantized in the non-scaling regime and the entropy was determined to grow as $S \sim N^{2 / 3}$. The non-scaling regime is characterized by $N<I / 2$ whereas scaling solutions satisfy $N>I / 2$. We remind the reader that scaling solutions are solutions where the centers, which are labelled by points $x \in \mathbf{R}^{3}$, can approach each other arbitrarily closely (see section 2.1.2).

Earlier arguments in the literature [17, 20-22] have suggested that scaling solutions carry vastly more entropy and may account for a large fraction of the black hole entropy. Here we will see that this is not the case, at least for this large class of solutions. However, as we will point out, the (leading) entropy coming from these solutions matches that of free gravitons in $\mathrm{AdS}_{3} \times \mathrm{S}^{2}$. The change in the leading degeneracy between the non-scaling and scaling regime seems to precisely take into account the stringy exclusion principle [8], which for a chiral primary in the NS sector states that $\tilde{L}_{0} \leq c / 12$.

\subsection{The dipole halo solution space}

In this section we will review some essential formulas and results on the multicenter solutions of our interest. We refer readers unfamiliar with the subject to the earlier literature $[2,4-7,23,24]$. As was discussed in $[2,17]$ this system manifests two different behaviors depending on the value of the D0 charge. ${ }^{4}$

\subsubsection{Charges and constraints}

To be more precise let us first specify what we mean by the charges D6 and $\overline{\mathrm{D} 6}$. These are D6 branes wrapping the entire Calabi-Yau which carry lower dimensional brane charge induced entirely from a non-trivial worldvolume Abelian flux, $F^{A}=p^{A} / 2$, on the D6 and

\footnotetext{
${ }^{4}$ Throughout this note we are going to be sloppy and use D0 instead of $\overline{\mathrm{D} 0}$ for brevity.
} 
the opposite flux on the $\overline{\mathrm{D} 6}$. Explicitly, the full charge vector including D6, D4, D2 and D0 charge, in the same notation as [2], reads:

- D6, $\Gamma_{6}=\left(1, \frac{1}{2} p^{A}, \frac{1}{8} D_{A B C} p^{B} p^{C}, \frac{1}{48} D_{A B C} p^{A} p^{B} p^{C}\right)=\left(1, \frac{p}{2}, \frac{p^{2}}{8}, \frac{p^{3}}{48}\right)$.

- $\overline{\mathrm{D} 6}, \Gamma_{\overline{6}}=\left(-1, \frac{1}{2} p^{A},-\frac{1}{8} D_{A B C} p^{B} p^{C}, \frac{1}{48} D_{A B C} p^{A} p^{B} p^{C}\right)=\left(-1, \frac{p}{2},-\frac{p^{2}}{8}, \frac{p^{3}}{48}\right)$.

where $D_{A B C}$ is the triple intersection of a basis of four cycles in the Calabi-Yau, and we suppressed some subleading contributions which depend on the second Chern class of the Calabi-Yau. In addition to the D6 $\overline{\mathrm{D} 6}$ pair, the system consists of an arbitrary number, $n$, of D0's of charge $\Gamma_{a}=\left\{0,0,0,-q_{a}\right\}$ with all the $q_{a}$ positive and $\sum_{a} q_{a}=N$, bound to a D6 and $\overline{\mathrm{D} 6}$.

To specify the full supergravity solution corresponding to backreacting these branes it is necessary to fix the moduli at infinity and also specify the locations of the branes (or centers as we refer to them in supergravity) which are given by vectors, $\vec{x}_{6}, \vec{x}_{\overline{6}}, \vec{x}_{a} \in \mathbb{R}^{3}$, in the non-compact spatial directions. Throughout these notes we will often find it convenient to work with asymptotically $\mathrm{AdS}_{3} \times \mathrm{S}^{2}$ solutions and thus we will work in the decoupling limit of [7] which fixes the asymptotic Kähler moduli at $p^{A} \infty$ (which in this specific case is also a "threshold point" [7]).

The locations of the centers are not entirely free but must satisfy so-called "integrability conditions" [4]. It is precisely these conditions that make the solution space a topologically interesting manifold. In this case, where we have assumed AdS asymptotics, they take the form

$$
\begin{gathered}
-\frac{q_{a}}{x_{6 a}}+\frac{q_{a}}{x_{\overline{6} a}}=0 \\
-\frac{I}{x_{6 \overline{6}}}+\sum_{a} \frac{q_{a}}{x_{6 a}}=-\beta
\end{gathered}
$$

Here $I=-\left\langle\Gamma_{6}, \Gamma_{\overline{6}}\right\rangle=\frac{p^{3}}{6}$ is given in terms of the total D4-charge $p^{A}$ of the system and $\beta=\left\langle\Gamma_{6}, h\right\rangle$ with $I, \beta>0$. Here $\langle-,-\rangle$ is an anti-symmetric intersection product on the charge space and $h$ is a vector (in the charge lattice) specifying the asymptotics of the solution, see e.g. [4]. It is clear from the first line that the D0's are forced to lie in the plane equidistant from the $D 6$ and $\overline{D 6}$, as we are at threshold, and so we can simply write $x_{a}:=x_{6 a}=x_{\overline{6} a}$.

The integrability equations alone are in principle not sufficient to guarantee the existence of a well-defined solution. For non-scaling solutions, there are strong arguments [23] that existence of the solution is equivalent to the existence of a so-called attractor flow tree, which does indeed exist for the D6 $\overline{\mathrm{D} 6} \mathrm{D} 0$ solutions [7, 21]. For scaling solutions this argument does not apply, but for the case at hand the main difference between scaling and non-scaling solutions lies in the existence of solutions which resemble the D0-D4 black hole very closely. As the latter have no pathologies, we expect that for $\mathrm{D} 6 \overline{\mathrm{D} 6} \mathrm{D} 0$ systems the integrability conditions are equivalent to the existence of a solution, but it would be nice to separate check of this. 


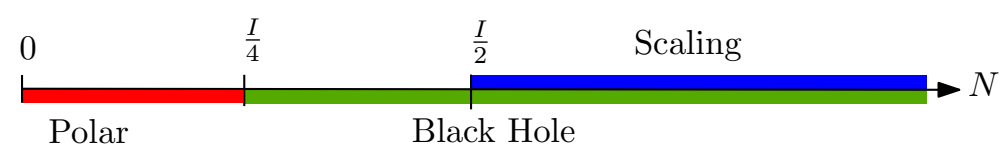

Figure 1. We show the different physical regimes of the D6 $\overline{\mathrm{D} 6} \mathrm{D} 0$ system in terms of the total charge carried by the $n$ D0s, $N$. Note that the total D0-charge is $q_{0}=\frac{I}{4}-N$ with $I=\frac{p^{3}}{6}$. The polar regime corresponds to total charges for which no single center D4D0 black hole exists while in the black hole regime there exists a D4D0 black hole with the same total charges as the D6 $\overline{\mathrm{D} 6} \mathrm{D} 0$ system. Another regime is what we call the 'scaling' regime in which the D6 $\overline{\mathrm{D} 6} \mathrm{D} 0$ solution space contains a point where the different centers become coincident.

\subsubsection{Scaling solutions}

The two different regimes mentioned above are the non-scaling case $(N<I / 2)$ which was discussed in detail in section 6 of [2] and the scaling regime $(N \geq I / 2)$, within which the centers can approach each other arbitrarily closely. In figure 1 we show how the scaling and non-scaling regimes overlap with the so called polar and black hole regimes. Notice that the scaling solutions we consider do not cover the region with $I / 4<N<I / 2$, while black holes exist in this regime. It is conceivable that other scaling solutions exist, e.g. with different D6-charges or more than two centers with D6 charge, which cover this regime, and it would be interesting to explore this further.

To see that in the scaling regime the centers can approach each other, let us place all the D0 charge at one center so $q_{1}=N$ and consider solutions of the form

$$
x_{6 \overline{6}}=\lambda I+\mathcal{O}\left(\lambda^{2}\right) \quad x_{61}=\lambda N+\mathcal{O}\left(\lambda^{2}\right)
$$

For small $\lambda$ solutions of this form can always be found so long as $N \geq I / 2$; the latter requirement coming from the fact that $x_{6 \overline{6}}$ and $x_{61}$ are coordinate separations and must satisfy triangle inequalities. As $\lambda \rightarrow 0$ the coordinate distance between the centers goes to zero and the centers coincide in coordinate space. In physical space, however, warp factors in the metric blow up generating a deep throat that keeps the centers a fixed metric distance apart even as $\lambda \rightarrow 0$. Outside of this arbitrarily deep throat the solutions is almost indistinguishable from a D4D0 black hole. This regime is thus of great physical relevance and e.g. in [17] it was conjectured to correspond to the deconstruction of a D4D0 black hole.

\subsubsection{Symplectic form}

We are now ready to review the construction of the associated symplectic form. Recall [2] that once the symplectic form on the solution space (parameterized by the locations of centers satisfying (2.1)-(2.2)) has been found it can be used to quantize the system using methods of geometric quantization. To make this paper somewhat self-contained we begin by reproducing the relevant part of section 6 of [2]. For a derivation and more background the reader is referred to [2].

An explicit expression for the symplectic form can be obtained using the following coordinate system [2]. We define an orthonormal frame $(\hat{u}, \hat{v}, \hat{w})$ fixed to the D6 $\overline{\mathrm{D} 6}$ pair, 


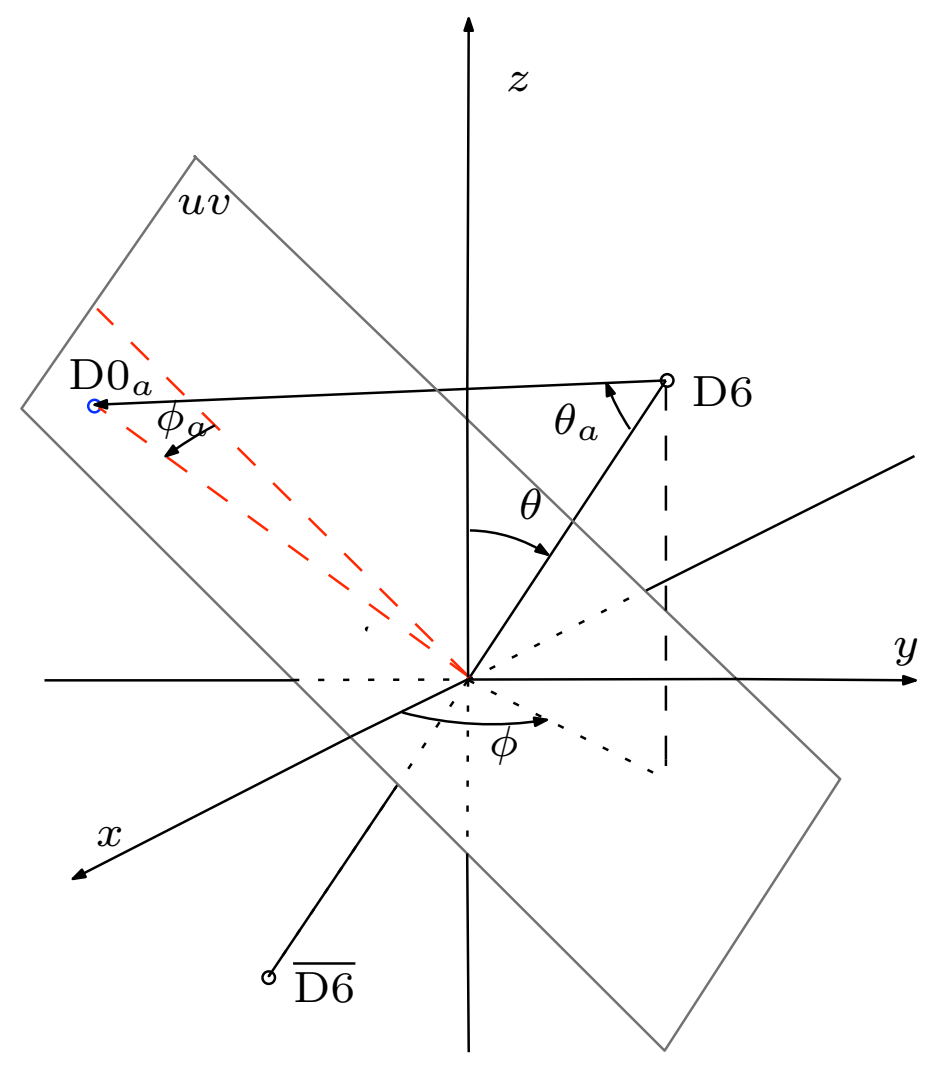

Figure 2. The coordinate system used to derive the D6 $\overline{\mathrm{D} 6}-\mathrm{N}$ D0 symplectic form. The coordinates $(\theta, \phi)$ define the orientation of the $(\hat{u}, \hat{v}, \hat{w})$ axis with respect to the fixed, reference, $(\hat{x}, \hat{y}, \hat{z})$ axis. The $\mathrm{D} 6 \overline{\mathrm{D} 6}$ lie along the $\hat{w}$ axis (with the origin between them) and the D0's lie on the $\hat{u}-\hat{v}$ plane at an angle $\phi_{a}$ from the $\hat{u}$-axis. The radial position of each D0 in the $\hat{u}-\hat{v}$ plane is encoded in the angle $\theta_{a}$ (between $\vec{x}_{6 \overline{6}}$ and $\vec{x}_{6 a}$ ).

such that the D6 $\overline{\mathrm{D} 6}$ lie along the $w$ axis and with the D0's lying in the $u-v$ plane. Rotations of the system can then be interpreted as rotations of the $(\hat{u}, \hat{v}, \hat{w})$ frame with respect to a fixed $(\hat{x}, \hat{y}, \hat{z})$ frame. We will parameterize the choice of $\hat{w}$-axis in the standard fashion by two angles, $(\theta, \phi)$. We can furthermore specify the location of the $a^{\prime}$ th D0 with respect to D6 $\overline{\mathrm{D} 6}$ pair by two additional angles, $\left(\theta_{a}, \phi_{a}\right)$. The first angle, $\theta_{a}$, is the one between $\vec{x}_{6 \overline{6}}$ and $\vec{x}_{6 a}$, while $\phi_{a}$ is a polar angle in the $u$-v plane. Our $2 n+2$ independent coordinates on solution space are thus $\left\{\theta, \phi, \theta_{1}, \phi_{1}, \ldots, \theta_{n}, \phi_{n}\right\}$. This coordinate system is depicted in figure 2 .

The standard Euclidean coordinates of the centers are then given in terms of the angular coordinates by

$$
\begin{array}{ll}
\vec{x}_{6}=\frac{j}{\beta} \hat{w} & \hat{u}=\cos \phi \hat{x}-\sin \phi \hat{y} \\
\vec{x}_{\overline{6}}=-\frac{j}{\beta} \hat{w} & \hat{v}=\sin \phi \cos \theta \hat{x}+\cos \phi \cos \theta \hat{y}-\sin \theta \hat{z} \\
\vec{x}_{a}=\frac{j \tan \theta_{a}}{\beta}\left(\cos \phi_{a} \hat{u}+\sin \phi_{a} \hat{v}\right) & \hat{w}=\sin \phi \sin \theta \hat{x}+\cos \phi \sin \theta \hat{y}+\cos \theta \hat{z}
\end{array}
$$


The angular momentum, $j\left(\theta_{a}\right)$, is a function of the other coordinates rather than an independent coordinate, and is given by

$$
j=\frac{I}{2}-\sum_{a} q_{a} \cos \theta_{a} .
$$

Using this explicit coordinatization the symplectic form turns out to be [2]:

$$
\Omega=-\frac{1}{4} d\left[2 j \cos \theta d \phi+2 \sum_{a} q_{a} \cos \theta_{a} d \phi_{a}\right]
$$

with $d$ denoting the exterior derivative.

The symplectic form (2.8) is non-degenerate on the BPS solution space parameterized by the locations of the centers implying that the latter is in fact a phase space. By virtue of arguments in [2] this space can be quantized in its own right, ignoring the much larger non-BPS solution space in which it is embedded, and from this treatment one might hope to extract information about the BPS states of the full theory (including at least the number of such states).

Note that, as is manifest from our angular coordinatization, the phase space is actually toric with a $\mathrm{U}(1)^{n+1}$ action coming from $\phi$ and the $n \phi_{a}$ 's. This is a consequence of the fact that the D0's are mutually non-interacting; their sole interaction is via the D6 $\overline{\mathrm{D} 6}$. This toric structure greatly simplifies the quantization of the solution and was essential in [2].

\subsubsection{Physical picture}

As much of the subsequent presentation will be a rather technical treatment of the phase space we would like to lend the reader some intuition. We begin by recalling [4] that the angular momentum carried by these solutions is

$$
\vec{J}=\sum_{i<j} \vec{J}_{i j}, \quad \vec{J}_{i j}:=\frac{\left\langle\Gamma_{i}, \Gamma_{j}\right\rangle \vec{x}_{i j}}{2 r_{i j}}
$$

where now $i, j$ run over all centers, including the D6s. Thus each pair of centers contributes angular momentum $\vec{J}_{i j}$ to the total. The length of these vectors is fixed to $\left\langle\Gamma_{i}, \Gamma_{j}\right\rangle / 2$ but their direction is not fixed. The dependence on the intersction product $\left\langle\Gamma_{i}, \Gamma_{j}\right\rangle$, pairing electric and magenetic sources, reflects the fact that this angular momentum is carried by the electromagnetic field and is due to crossed electric and magnetic fields. Since the D0's have vanishing intersection product with each other there are only $(2 n+1)$ momenta vectors: $\vec{J}_{6 \overline{6}}, \vec{J}_{6 a}$, and $\vec{J}_{\overline{6} a}$.

As we will see, our quantization can essentially be understood as quantizing the direction of these vectors, or more precisely the size of their projection on a given "z-axis", yielding familiar angular momentum multiplets. Naively the phase space of these angular momentum vectors is the direct product of $(2 n+1)$ two-spheres and the number of states is just the product of the factors $\left(2\left|\vec{J}_{i j}\right|+1\right)$ from each multiplet. The geometric origin of the momenta (i.e. endpoint of multiple vectors fixed to be the same center), however, as well as the constraint equations (2.1)-(2.2) fix the possible relative orientations of the different 


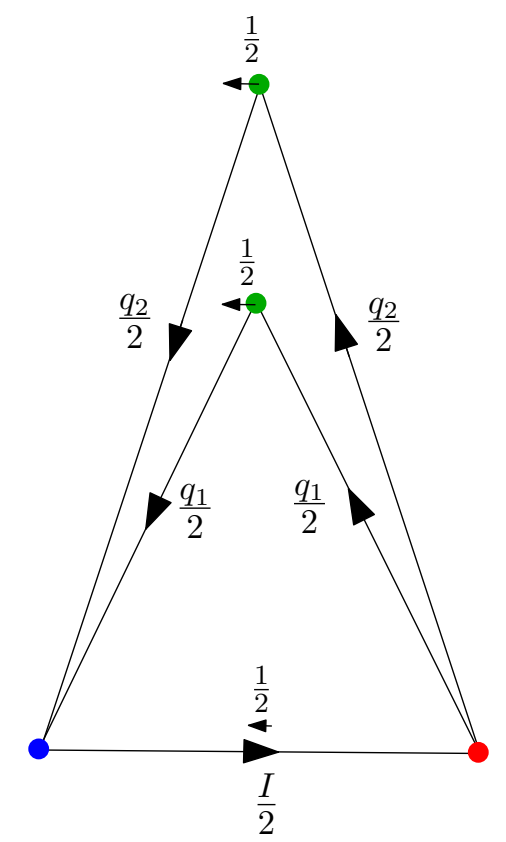

Figure 3. In this figure all contributions to the total angular momentum are shown. The large arrows denote the classical angular momenta carried by the electromagnetic field. They are proportional to the intersection products of the charges, D6 (red), $\overline{\mathrm{D} 6}$ (blue) and D0 (green). The small arrows extending from the D0's represent their spin aligning with the dipole magnetic field sourced by the D6 $\overline{\mathrm{D} 6}$-pair, the small arrow in the bottom is the spin of the center of mass multiplet of the D6 $\overline{\mathrm{D} 6}$-pair aligning with the magnetic fields.

angular momentum vectors. As a result not all states of the full free angular momentum multiplets are allowed. Rather, the correct phase space is now a more complicated fibration of spheres of varying size and, although intuitively it is still insightful to think of the states as part of "angular momentum multiplets", they now only fill out a constrained subspace of the product of the full multiplets. For instance, since $\vec{J}_{6 a}$ and $\vec{J}_{\overline{6} a}$ always end at the same point, their orientation relative to the $w$-axis is not independent so, rather than two angular momentum multiplets, these vectors yield only a single multiplet (the diagonal multiplet in their free product).

The best way to get some intuition for this is to consider the symplectic form on the phase space of our system. Using the coordinate system (2.6) and introducing the notation

$$
\begin{aligned}
\vec{J} & =J_{z} \hat{z}+J_{y} \hat{y}+J_{x} \hat{x} \\
\vec{J}_{6 a} & =\vec{J}^{a}=J_{w}^{a} \hat{w}+J_{v}^{a} \hat{v}+J_{u}^{a} \hat{u} \\
\vec{J}_{\overline{6} a} & =\vec{J}^{a}=J_{w}^{\bar{a}} \hat{w}+J_{v}^{\bar{a}} \hat{v}+J_{u}^{\bar{a}} \hat{u}
\end{aligned}
$$

we can cast the dipole halo symplectic form in a more suggestive form

$$
\Omega=-\frac{1}{2}\left[d J_{z} \wedge d \phi+\sum_{a} d J_{w}^{a} \wedge d \sigma_{a}+\sum_{a} d J_{w}^{\bar{a}} \wedge d \sigma_{\bar{a}}\right]
$$


with $\left|\overrightarrow{J^{a}}\right|=\left|\overrightarrow{J^{a}}\right|=q_{a} / 2$. Because $\vec{J}^{a}$ and $\overrightarrow{J^{a}}$ are related by the location of the D0 they end on the last two terms above can be combined yielding

$$
\Omega=-\frac{1}{2}\left[d J_{z} \wedge d \phi+2 \sum_{a} d J_{w}^{a} \wedge d \sigma_{a}\right] .
$$

If there were no other constraints, the $J_{w}^{a}$ would independently be able to take values between $\pm\left|\overrightarrow{J^{a}}\right|$. However, as we will discuss below, they have to satisfy the bounds $J_{w}^{a}>0$ and $2 \sum_{a} J_{w}^{a} \leq I / 2$, leading to a more intricate phase space with a Hilbert space that is no longer a product of "free" angular momentum multiplets. There is also another angular momentum multiplet, coming from the total angular momentum $\vec{J}$, and this gives rise to a full multiplet with $-|J|<J_{z}<|J|$. The size of $\vec{J}$, however, depends on the $J^{a}$ (even classically). Thus, each state in the Hilbert state labelled by $J^{a}$ quantum numbers will be tensored with a $J$ multiplet corresponding to the total $\vec{J}$ associated to its $J^{a}$ quantum numbers (via $J=I / 2-2 \sum_{a} J_{w}^{a}$ ).

This is the intuitive physical picture for which we develop a precise mathematical treatment in the next subsection, using the observation of [2] that the phase space is a toric manifold. The upshot is, however, that we are doing nothing more than quantizing angular momentum variables, but ones that are non-trivially connected and constrained.

There is a second physical phenomena that only appears when quantizing the system, which we would like to highlight here. As stressed above, we are in essence quantizing the classical angular momentum of the system. However, when we quantize we need to take into account the intrinsic spin of the particles involved, as was beautifully explained in [24]. As pointed out there, the centers are superparticles containing excitations in various spin states. Due to the presence of magnetic fields, however, the lowest energy BPS state is a spin half state, where energy is gained by aligning the intrinsic magnetic dipole moment with the magnetic field. The situation is sketched for our dipole halo system in figure 3. Including these quantumcorrections the size of the total angular momentum is given by

$$
J=\frac{I-1}{2}-\sum_{a}\left(q_{a} \cos \theta_{a}+\frac{1}{2}\right) .
$$

These spins are especially important when considering classical scaling solutions, as we discuss further in section 3.1.

\subsection{The dipole halo states}

As mentioned earlier the system under study manifests two regimes. The non-scaling one $(N<I / 2)$ was studied in detail in [2], and here we will extend that calculation so that the whole allowed range, $N>0$, is covered.

In this section we will count states using techniques of geometric quantization of the supergravity solution spaces developed in [2]; in the next section we will compare this to the calculation of free supergravity states on $\mathrm{AdS}_{3}$ and see that the two results agree beautifully. 


\subsubsection{States and polytopes}

In our previous work [2] we showed that for the D6 $\overline{\mathrm{D} 6} \mathrm{D} 0$ system the solution space is a toric manifold. This allowed us to construct all the normalizable quantum states explicitly. In this paper we will be less interested in the explicit form of the wave-functions than in their number. In appendix A we show how the number of states can be easily obtained from the combinatorics of the toric polytope. We will not review the technology of geometric quantization of toric manifolds here but instead refer the reader to appendix B of [2] and appendix A of this note.

From the symplectic form (2.8) we read off the coordinates on the polytope

$$
y=j \cos \theta, \quad y_{a}=q_{a} \cos \theta_{a} \geq 0
$$

So we see that the polytope is bounded by the inequalities

$$
-j \leq y \leq j, \quad 0 \leq y_{a} \leq q_{a}
$$

and furthermore the requirement that the angular momentum is positive

$$
j=\frac{I}{2}-\sum_{a} y_{a} \geq 0 .
$$

It is this last condition that differentiates the non-scaling regime $N=\sum_{a} q_{a}<I / 2$ from the scaling regime $N \geq I / 2$. In the former range the condition (2.18) is redundant in the definition of the polytope as it is automatically satisfied for all values of $x_{a}$ allowed by the other constraints (2.17). In case $N>I / 2$ the constraint (2.18) actually becomes essential and can make some of the constraints (2.17) redundant, although this depends on the specific values of the $q_{a}$. What is shared by all the solution spaces in the scaling case is that it is possible to approach the point where all centers coincide arbitrarily closely, which automatically implies that $j$ has to approach zero. When this happens, an infinitely deep scaling throat forms in space-time [20, 24]. For more than a single D0 center there are however different types of solution spaces with a scaling point, depending on the specific values of the charges $q_{a}$. We show all the different possible polytope topologies for the case with two D0 centers in figure 4, clearly the number of topologies grows very fast with the number of D0-centers.

Given the defining inequalities (2.17) and (2.18) we can use eq. (A.6) from the appendix (see also the example containing eq. (A.2)) to see that there is a unique quantum state corresponding to each set of integers $\left(m, m^{a}\right)$ satisfying

$$
\begin{array}{r}
0 \leq m^{a} \leq q_{a}-1, \quad \sum_{a}\left(m^{a}+\frac{1}{2}\right) \leq \frac{I-1}{2}, \\
-\left[\frac{I-1}{2}-\sum_{a}\left(m^{a}+\frac{1}{2}\right)\right] \leq m+\frac{1}{2} \leq\left[\frac{I-1}{2}-\sum_{a}\left(m^{a}+\frac{1}{2}\right)\right]
\end{array}
$$

The $\left(m, m_{a}\right)$ above are simply quantized angular momenta corresponding to quantizing the angles $\left(\theta, \theta_{a}\right)$ appearing in $(2.17)-(2.18)$. The half-integral shifts are related to the 

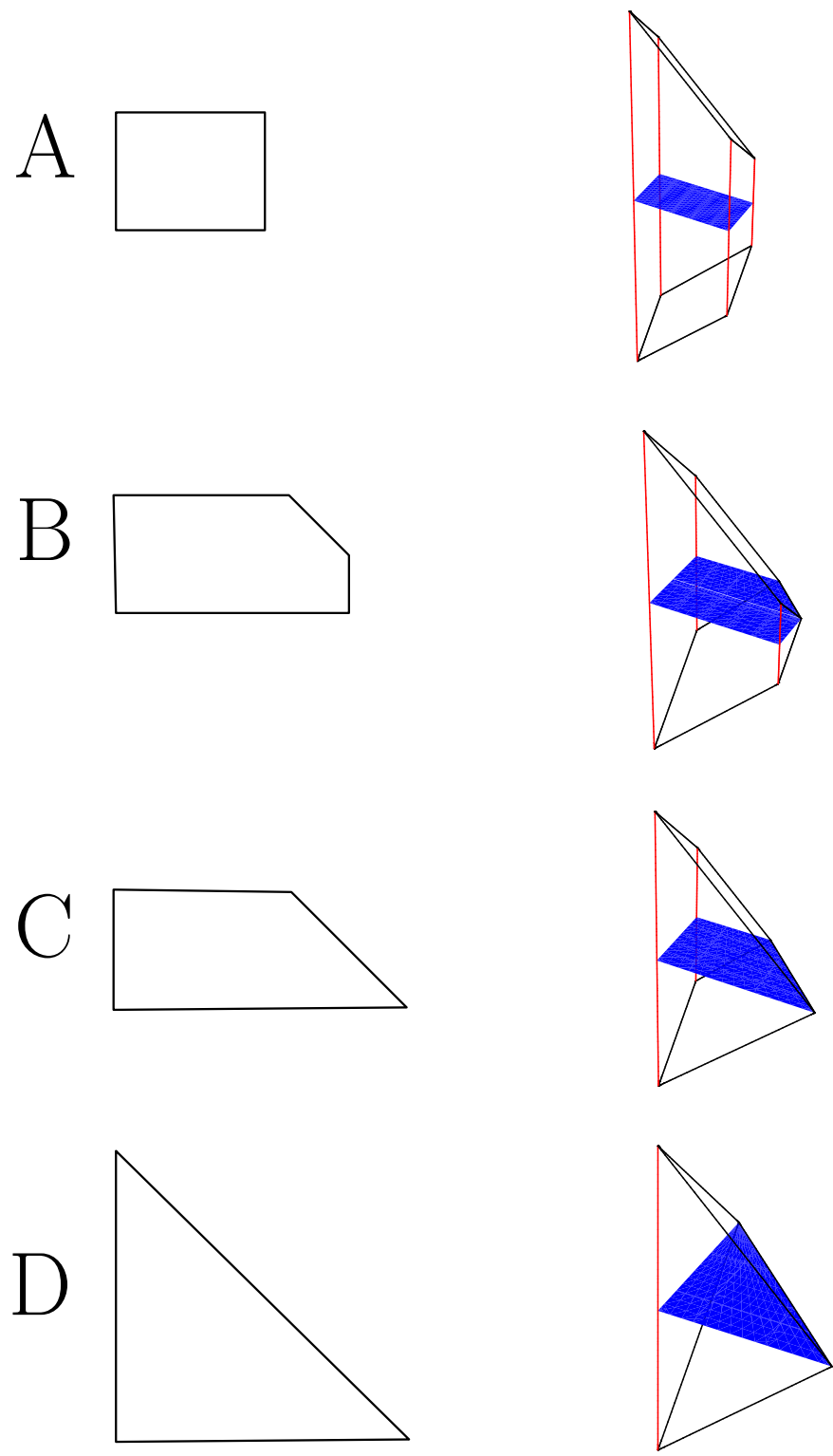

Figure 4. These are the different types of polytopes corresponding to a D6 $\overline{\mathrm{D} 6} \mathrm{D} 0$ system with 4 centers. On the left the 'base' polytope determined by the coordinates $y_{1}$ and $y_{2}$ with $y=0$ is shown, on the right also the fiber spanned by the coordinate $y$ is included, the edges along this direction are drawn in red, the surface $y=0$ is shown in blue. See (2.16) for a definition of the coordinates. The different cases correspond respectively to: Case A (non-scaling) $q_{1}+q_{2}<\frac{I}{2}$, Case B (scaling) $q_{1}+q_{2} \geq \frac{I}{2}$ and $q_{1}, q_{2} \leq \frac{I}{2}$, Case C (scaling) $q_{2} \leq \frac{I}{2} \leq q_{1}$, and Case D (scaling) $\frac{I}{2} \leq q_{1}, q_{2}$. So we see that from 4 centers onward there are different types of scaling polytopes, a feature that was absent for three scaling centers.

fermionic nature of the centers as discussed in $[2,24]$ and the coupling to the extrinsic spin, as explained at the end of section 2.1.4.

To be precise the constraints above only hold under the assumption that all D0 centers carry different charges, $q_{a}$. To relax this assumption we introduce integer multiplicities, 
$n_{a}$, for each charge $q_{a}$ so that $N=\sum_{a} n_{a} q_{a}$ and $n=\sum_{a} n_{a}$. We now have to take into account the quantum indistinguishability of these (fermionic) particles. As explained in detail in [2] this translates to taking the appropriate orbifold of the polytope or, in terms of (2.20), augmenting the $m^{a}$ by an additional label $i_{a}$ running from $1, \ldots, n_{a}$ and requiring them to satisfy

$$
\begin{gathered}
0 \leq m_{1}^{a}<m_{2}^{a}<\ldots<m_{n_{a}}^{a}<q_{a}, \quad \sum_{a, i}\left(m_{i_{a}}^{a}+\frac{1}{2}\right) \leq \frac{I-1}{2} \\
-\left[\frac{I-1}{2}-\sum_{a, i}\left(m_{i_{a}}^{a}+\frac{1}{2}\right)\right] \leq m+\frac{1}{2} \leq\left[\frac{I-1}{2}-\sum_{a, i}\left(m_{i_{a}}^{a}+\frac{1}{2}\right)\right]
\end{gathered}
$$

These constraints are fermionic, enforcing Pauli exclusion of indistinguishable centers. Note also that they reduce to $(2.20)$ if all the $n_{a}=1$.

\subsubsection{The D6 $\overline{\mathrm{D} 6} \mathrm{D} 0$ partition function}

In this section we will count the combined number of supergravity states $d_{N}$ of all D6 $\overline{\mathrm{D} 6} \mathrm{D} 0$ systems with total charge $\left(p^{A}, \frac{p^{3}}{24}-N\right)$. More precisely we will calculate the leading term of $S(N)=\log d_{N}$ in a large $N$ expansion. We will notice there are two phases depending on the relative value of $I=p^{3} / 6$ and $N$, separated by a transition at $N=I$. The first phase was already found in [2] where the counting was performed in the regime $N<I / 2$. For larger $N$ the appearance of scaling solutions slightly complicates the counting but can still be performed as shown below. What is interesting is that the existence of scaling solutions seems only to become dominant at $N=I$ where a phase transition occurs.

As we previously pointed out, in the scaling regime there is an additional constraint that complicates the polytope and makes the counting of integer points inside slightly more difficult. We will find it convenient not to calculate a fully explicit generating function $Z$, as we did before in [2]. Instead, since we are only interested in the large $N$ regime, it will be sufficient for us to find the leading term of $\log Z$ in a large $N$ expansion.

The complication in the scaling regime arises because of the second constraint in equation (2.21). To proceed let us introduce the quantity

$$
M=\sum_{a, i}\left(m_{i_{a}}^{a}+\frac{1}{2}\right),
$$

As the $m_{i_{a}}^{a}$ are the discrete analogues of the classical $q_{a} \cos \theta_{a}$ the interpretation of $M$ is as the amount of angular momentum carried by the D0 centers (which, by the integrability constraints $(2.1),(2.2)$, is always opposite in direction to the angular momentum carried by the $\mathrm{D} 6 \overline{\mathrm{D} 6}$ pair):

$$
M=\frac{I}{2}-\frac{1}{2}-J
$$

Both the $\frac{1}{2}$ in the above formula and in (2.23) arise due to the spin contributions to the quantum mechanical angular momentum (see the end of section 2.1.4 for a detailed explanation). 
Now if we succeed in calculating the degeneracy $d_{N, M}$ as a function of $M$, then the full degeneracy will be

$$
d_{N}=\sum_{M=1 / 2}^{(I-1) / 2} d_{N, M}
$$

The full degeneracy will clearly be less than $I / 2$ times $d_{N, M^{\prime}}$ where $M^{\prime}$ is the value of $M$ which maximizes $d_{N, M}$. Thus instead of calculating the sum it will be sufficient for us to find the $M^{\prime}$ that maximizes $d_{N, M}$ because

$$
S(N)=S\left(N, M^{\prime}\right)+\Delta S,
$$

where we have defined

$$
\Delta S=\log \sum_{M=1 / 2}^{(I-1) / 2} e^{S(N, M)} S\left(N, M^{\prime}\right) \leq \log I,
$$

so, as long as the leading entropy is a power-law (rather than a logarithm) in the charges, we can find the leading term in $S(N)$ by calculating $S(N, M)$ and maximizing over $M$.

As we will now show it is not too hard to calculate a generating function for $d_{N, M}$

$$
\mathcal{Z}(q, y)=\sum_{N, M}^{\infty} d_{N, M} q^{N} y^{M} .
$$

Note that this does not reduce to a generating function for $d_{N}$ by setting $y=1$ as in this generating function we sum over $M=1, \ldots, \infty$ while in the case of interest the range of $M$ is restricted.

Let us derive an expression for (2.28) by approximating it in a few steps. A first key ingredient is that for a partition of $N=\sum_{a} n_{a} q_{a}$, one has

$$
0 \leq m_{1}^{a}<\ldots<m_{n_{a}}^{a}<q_{a}, \quad M=\sum_{a, i}\left(m_{i}^{a}+\frac{1}{2}\right)
$$

Forgetting for the moment about $N$, the above relation is just a fermionic partition of $M$. This is given by

$$
\mathcal{Z}_{\text {ferm }}=\prod_{l \geq 1}\left(1+y^{l-1 / 2}\right)
$$

We need to reintroduce the information about $N$. To do so remember that the sole role of the partition of $N$ is to specify the number of $m_{i}^{a}$ above. Keeping this key point in mind we proceed in two steps. First assume that we have $n$ centers with the same charge $k$ only $(N=n k)$, then it is easy to see that the appropriate modification of $\mathcal{Z}_{\text {ferm }}(2.30)$ is

$$
\mathcal{Z}_{\text {int }}=\prod_{1 \leq l \leq k}\left(1+q^{k} y^{l-1 / 2}\right)
$$

this comes about because in expanding the expression above the number of centers in each term is simply the number of $q^{k}$ that appear in it. The product over possible $l$ is then 
a reflection of the constraint (2.29). Now to generalize to an arbitrary partition of $N$ we take a product of the above expression over all possible $k \geq 1$. This yields the core generating function

$$
\mathcal{Z}_{0}=\prod_{k \geq 1,1 \leq l \leq k}\left(1+q^{k} y^{l-1 / 2}\right)
$$

To get the actual generating function we include the contribution from $m$ in equation (2.22). The generating function is then

$$
\mathcal{Z}=\left(I-2 y \partial_{y}\right) \mathcal{Z}_{0}=\left(I-2 y \partial_{y}\right) \prod_{k \geq 1,1 \leq l \leq k}\left(1+q^{k} y^{l-1 / 2}\right)
$$

In evaluating the leading contribution to the entropy we can neglect the overall multiplicative factor because it will be subleading. Thus we focus on $\mathcal{Z}_{0}$.

\subsubsection{The entropy and a phase transition}

As is familiar from thermodynamics we can study the large energy regime by evaluating the partition function at large temperature. We introduce the potentials $\beta$ and $\mu$ through

$$
q=e^{-\beta}, \quad y=e^{-\mu}
$$

and can then look for the behavior of the entropy for $\beta, \mu \ll 1$.

$$
\begin{aligned}
\log \mathcal{Z}_{0} & =\sum_{k \geq 1,1 \leq l \leq k} \log \left(1+q^{k} y^{l-1 / 2}\right) \\
& =\sum_{n \geq 1}\left(\frac{(-1)^{n+1}}{n}\left[\sum_{k \geq 1} q^{n k}\left(\sum_{l=1}^{k} y^{n(l-1 / 2)}\right)\right]\right) \\
& =\sum_{n \geq 1}\left(\frac{(-1)^{n+1}}{n} \frac{y^{n / 2}}{1-y^{n}}\left[\sum_{k \geq 1} q^{n k}\left(1-y^{n k}\right)\right]\right) \\
& =\sum_{n \geq 1}\left(\frac{(-1)^{n+1}}{n} \frac{q^{n} y^{n / 2}}{\left(1-q^{n}\right)\left(1-q^{n} y^{n}\right)}\right) \\
& \sim\left(\sum_{n>1} \frac{(-1)^{n+1}}{n^{3}}\right) \frac{1}{\beta(\mu+\beta)}=: \frac{\alpha}{\beta(\mu+\beta)}
\end{aligned}
$$

with $\alpha=\frac{3}{4} \zeta(3)$. Using the above relation we find

$$
\begin{aligned}
& N=-\partial_{\beta} \log \mathcal{Z}_{0} \sim \frac{\alpha(\mu+2 \beta)}{\beta^{2}(\mu+\beta)^{2}} \\
& M=-\partial_{\mu} \log \mathcal{Z}_{0} \sim \frac{\alpha}{\beta(\mu+\beta)^{2}}
\end{aligned}
$$

From the equations above it follows that the approximation is valid for $N, M \gg 1$, which is exactly the regime we are interested in. Furthermore the relative size between $M$ and $N$ is determined by the ratio $\mu / \beta$ as

$$
N / M=2+\frac{\mu}{\beta} .
$$


The entropy in the large $M, N$ regime then reads

$$
S(N, M)=-\log \mathcal{Z}_{0}+\beta N+\mu M \sim \frac{\alpha}{\beta(\mu+\beta)} \sim(\alpha M[N-M])^{1 / 3} .
$$

Maximizing $S(N, M)$ over $M$ in the range ${ }^{5} 1 / 2<M<I / 2$ we find that

$$
S(N)= \begin{cases}\left(\alpha \frac{N^{2}}{4}\right)^{1 / 3} & \text { if } N \leq I \\ \left(\alpha \frac{I}{2}\left(N-\frac{I}{2}\right)\right)^{1 / 3} & \text { if } I \leq N\end{cases}
$$

The most entropic configuration always has $M^{\prime}=N / 2$ until $N=I$ and then the bound (2.18) restricts $M^{\prime}=I / 2$. Thus most entropy is realized by low angular momentum states (remember $J \sim I / 2-M$ ) and, deep in the scaling regime where $N>I$, most of the entropy is given by the $j=0$ states.

The saddle point approximation used to obtain eq. (2.39) is only valid for charges $N \lesssim I^{2}$ because the discussion above shows we are interested in $M \approx \frac{I}{2}$ and in that regime $N \gtrsim I^{2}$ is not consistent with $\mu, \beta \ll 1$, as can be seen from (2.35)-(2.36). We will presently focus on the regime $N \gg I$ which is still consistent so long as their ratio does not scale with $I$ in the large charge regime one considers.

For $N \gg I$ Cardy's formula implies the leading entropy of the associated black hole grows as [1]

$$
S_{\mathrm{BH}}(N, I) \sim 4 \pi \sqrt{\frac{N I}{4}}
$$

Thus the D6 $\overline{\mathrm{D} 6} \mathrm{D} 0$ configurations we are considering do not exhibit the correct growth of entropy as a function of the charges to dominate the black hole ensemble, especially for large charges they are parametrically subleading.

Associated with the change from the first to the second line of (2.39) appears to be a phase transition occurring at $N=I$. In this phase transition we seem to move from an asymmetric phase, $\langle j\rangle \neq 0$, to a symmetric phase $\langle j\rangle=0$, note that this transition is not $C^{\infty}$ but still continuous. It is not immediately clear that any physical meaning should be ascribed to this "phase transition" since these configurations are not the dominant constituents of this sector of the BPS Hilbert space. Curiously, however, this seems to mirror the phase transition of [7]. Although the latter was analyzed for different constituents centers, if we simply equate the total charges of the two systems then the critical point of [7] would be at $N \approx I / 4$ and would correspond to a transition from a phase with $\langle j\rangle \neq 0$ to a $\langle j\rangle=0$ phase as $N$ increases (note that here there is a discontinuous jump in $\langle j\rangle$ ). It is both curious and interesting that the set of states we obtained, while relatively sparse in the overall Hilbert space, nonetheless exhibits a non-trivial phase structure that even seems to qualitatively share some of the structure of the full theory.

In the regime $N \gg I$ of [1], the number of states we obtained was substantially smaller than total number of BPS states of the conformal field theory. One may therefore wonder whether other solutions of supergravity exist with the same asymptotic charges and which

\footnotetext{
${ }^{5}$ Note that we are interested in the large charge limit $I \gg 1$, so throughout the paper we will often neglect quantum mechanical shifts of $1 / 2$ to $I$
} 
could account for the missing states, or whether this is the best supergravity can do. Such additional solutions could look like complicated multi-centered solutions of the type we have been considering, or be of an entirely different form. To address this question we will now compute the spectrum and degeneracy of a gas of free supergravitons in $\operatorname{AdS}^{3} \times \mathrm{S}^{2}$. As we will argue, this will provide an estimate for the maximal number of states we might expect to be obtainable from supergravity. It turns out that this computation yields a result whose asymptotic expansion agrees precisely with the number of D6 $\overline{\mathrm{D} 6} \mathrm{D} 0$ states, which supports the claim that the supergravity does not give rise to significantly more states in addition to those that we described.

\subsection{Free supergravity estimate}

In the previous section we calculated the number of BPS states in a given D4D0-charge sector that can be associated to configurational degrees of freedom of a D6 $\overline{\mathrm{D} 6} \mathrm{D} 0$ system of that same total charge. As we pointed out, there is an exponential number of states leading to a macroscopic statistical entropy. However the entropy scales with a different power of the charges than the D4D0 black hole entropy, making it parametrically subleading in the large charge supergravity limit. In other words, although we found very many D6 $\overline{\mathrm{D} 6} \mathrm{D} 0$ states the corresponding single center black hole still has exponentially more of them, indicating that these are not generic states of the black hole.

One might still wonder, however, if this is due to our restriction to a specific set of smooth multicenter solutions and if perhaps a larger number of states can be found by quantizing more complicated multicentered configurations. In this section we will give some non-trivial evidence that this is not the case and that the black hole degrees of freedom have to be sought outside of supergravity. An example of such states could be those of the proposal $[15,17,18,25]$ or the possibly related setup of $[14,26]$. Roughly speaking the degrees of freedom in these pictures seem to reside in non-abelian D-brane degrees of freedom; see also [27].

The approach we take to get a "bound" on the degrees of freedom coming from supergravity states is to exploit the fact that both the D4D0 black hole and the D6 $\overline{\mathrm{D} 6} \mathrm{D} 0$ system (and its generalizations) can be studied in asymptotically AdS space via the decoupling limit of [7]. In this context, the counting of the previous section corresponds to counting backreacted supergravity solutions with the same asymptotics as the D4D0 BTZ black hole, whereas here we will simply count free supergravity modes in empty AdS. The advantage of working in this limit, where the supergravity fields become free excitations around a fixed $\mathrm{AdS}_{3} \times \mathrm{S}^{2} \times \mathrm{CY}_{3}$ background, is that it becomes relatively easy to count them. Free supergravitons organize themselves in representations of the $(0,4)$ superconformal isometry algebra, and we merely need to determine the quantum numbers of the highest weights of the representations. This can be done following e.g. [28, 29] by performing a KK-reduction of eleven dimensional supergravity fields on the compact $\mathrm{S}^{2} \times \mathrm{CY}_{3}$ space ${ }^{6}$ to fields living on $\mathrm{AdS}_{3}$. The supergravity spectrum can then be determined using pure representation

\footnotetext{
${ }^{6}$ Note that we will assume the size of the $\mathrm{CY}_{3}$ to be much smaller than that of the $\mathrm{S}^{2}$ so that we will only consider the massless spectrum on the $\mathrm{CY}$, while keeping track of the full tower of massive harmonic modes on the sphere.
} 
theoretic methods, in terms of the massless field content of the KK reduction of M-theory on the Calabi-Yau manifold.

\subsubsection{Superconformal quantum numbers}

We want to compare the number of states we found by counting the possible configurational degrees of freedom of a D6 $\overline{\mathrm{D} 6} \mathrm{D} 0$ system to the number of chiral primaries given by KK reduction of $5 \mathrm{~d}$ supergravity in the free field limit. To make this comparison as clear as possible let us first translate the conserved four dimensional charges of the solutions, as presented in the previous section, to quantum numbers under the $(0,4)$ superconformal isometry algebra of the $\mathrm{AdS}_{3} \times \mathrm{S}^{2}$ background we consider here. Such a dictionary was derived in [7] and can be straightforwardly applied to the D6 $\overline{\mathrm{D} 6 \mathrm{D} 0}$ case. The map from supergravity to CFT quantum numbers is (recall that $c=6 I$ )

$$
L_{0}=N, \quad \tilde{L}_{0}=\frac{I}{4}, \quad J_{3}=-J .
$$

States with these quantum numbers are Ramond ground states, with minimum eigenvalues under $\tilde{L}_{0}$, as expected for BPS states. The calculation of the KK-spectrum on $\mathrm{AdS}_{3}$, however, is most naturally phrased in the NS sector and thus we would like to work in this sector. Thus we relate the charges (2.41) by spectral flow [30] in the right moving sector to the charges of the corresponding states in the NS-sector. Performing the spectral flow explicitly (see e.g [7] for some details) we find

$$
L_{0}=N, \quad \tilde{L}_{0}=\frac{I}{2}-J, \quad J_{3}=\frac{I}{2}-J .
$$

As expected the BPS states manifest themselves in the NS sector as chiral primaries, satisfying the condition $\bar{L}_{0}=J_{3}$. The well known unitarity bound [30] on the R-charge of chiral primaries implies a bound on the range of the $4 \mathrm{~d}$ angular momentum:

$$
0 \leq J \leq \frac{I}{2}
$$

From the results of the previous section it is clear that the $\mathrm{D} 6 \overline{\mathrm{D} 6} \mathrm{D} 0$ configurations satisfy this bound. This bound was first observed to have consequences for $\mathrm{AdS}_{3} / \mathrm{CFT}_{2}$ in [8], where it was called a stringy exclusion principle. As was argued there, it has to be imposed by hand on the free supergravity spectrum. What is perhaps surprising is that in the fully interacting supergravity theory the bound seems to emerge dynamically as it follows (at least for the $\mathrm{D} 6 \overline{\mathrm{D} 6} \mathrm{D} 0$ system) from the integrability equations (2.1) which are essentially a consequence of the BPS equations of motion. We have no solid proof of this, but we were unable to find other multicentered supergravity configurations that violate the bound, even with flat space asymptotics where there is no direct connection to the exclusion principle in the CFT.

It is interesting to note that by $(2.24)$ and (2.42) we see that for the D6 $\overline{\mathrm{D} 6} \mathrm{D} 0$ system $\tilde{L}_{0}=M+1 / 2$ and that indeed also the bound on $M$, observed in the previous section, follows directly from the unitarity bound discussed above. Using the identification of $M$ and $\tilde{L}_{0}$, we can write the following analogue of the generating function (2.28):

$$
\mathcal{Z}=\operatorname{Tr}_{\mathrm{NS}, \mathrm{BPS}}(-1)^{F} q^{L_{0}} y^{\tilde{L}_{0}-1 / 2}
$$




\begin{tabular}{|l|l|l|}
\hline 5d origin & number & $\{s, \tilde{h}\}$-towers \\
\hline hypermultiplets & $2 h^{1,2}+2$ & $\left\{\frac{1}{2}, \frac{1}{2}+m\right\}$ \\
vectormultiplets & $h^{1,1}-1$ & $\{0,1+m\}$ and $\{1, m\}$ \\
gravitymultiplet & 1 & $\{-1,2+m\},\{0,2+m\},\{1,1+m\}$ and $\{2,1+m\}$ \\
\hline
\end{tabular}

Table 1. Summary of the spectrum of chiral primaries on $\mathrm{AdS}_{3}$. The states are organized in towers of the form (2.45), the number of such towers and their characteristics are determined by the properties of the original theory and the details of the reduction. In the above table, $m$ is an arbitrary nonnegative integer.

Some remarks are in order. First we would like to point out that, for computational simplicity, we will calculate, in this section, an index rather than an absolute number of states, the difference with $(2.28)$ being an explicit insertion of $(-1)^{F}$. As one can see explicitly from the derivation below, the difference between the absolute number of states and the index will be only affect the numerical coefficient of the entropy, but not its functional dependence on the charges. Second, note that at $y=0$ the above index coincides with the standard elliptic genus for this theory.

\subsubsection{The spectrum of BPS states}

To calculate the degeneracies we are interested in, we need to enumerate the possible BPS states of linearized (free) supergravity on $\mathrm{AdS}_{3} \times \mathrm{S}^{2}$. It is often easier to enumerate these states via their quantum numbers in the CFT so we will use this language.

As we only have supersymmetry in the right moving sector, there are no BPS constraints on the left moving fields and thus all descendants of highest weight states will appear. The right-moving sector has $N=4$ supersymmetry and BPS states must be chiral primaries of a given weight. As a consequence, and as was shown in detail in e.g $[28,29,31,32]$, the full BPS spectrum can be written in the form: ${ }^{7}$

$$
\{s, \tilde{h}\}=\oplus_{n \geq 0}\left(L_{-1}\right)^{n}|\tilde{h}+s\rangle_{L} \otimes|\tilde{h}\rangle_{R}
$$

where $|h\rangle_{L}$ are highest weight states of weight $h$ of the left-moving Virasoro algebra and $|\tilde{h}\rangle_{R}$ are weight $\tilde{h}$ chiral primaries of the right-moving $\mathcal{N}=4$ super-Virasoro algebra.

Each field of five dimensional supergravity gives rise to a set of BPS states and their descendants after KK-reduction, where $\tilde{h}$ essentially labels the different spherical harmonics, while $n$ labels momentum excitations in $\mathrm{AdS}_{3}$ and $s$ the spin of the particle. It was shown in $[28,29,31,32]$ that, given the precise field content of $5 \mathrm{~d} \mathcal{N}=1$ supergravity, the reduction on a 2 -sphere gives the set of quantum numbers shown in table 1.

\footnotetext{
${ }^{7}$ Furthermore, to be fully precise we should point out that there remain so called singleton representations, but, for our purposes, we can ignore them as one can show they only contribute to subleading terms in the entropy in the large charge limit.
} 
Notice that the quantum numbers $\{s, \tilde{h}\}$ are of the form $\left\{s, \tilde{h}_{\min }+m\right\}$, and for each such set the partition function (2.44) has the following form

$$
Z_{\left\{s, \tilde{h}_{\min }\right\}}=\prod_{n \geq 0} \prod_{m \geq 0}\left(1-y^{m+\tilde{h}_{\min }-1 / 2} q^{n+m+\tilde{h}_{\min }+s}\right)^{(-1)^{2 s+1}}
$$

with the total partition function given by a product of such factors.

To extract the large $N$ degeneracies we proceed as in (2.34) and calculate the free energy corresponding to this partition function. We then evaluate it in the $\beta, \mu \ll 1$ limit $\left(q=e^{-\beta}, y=e^{-\mu}\right)$ :

$$
\begin{aligned}
F_{\left\{s, h_{\min }\right\}} & =(-1)^{2 s} \sum_{n \geq 1} \frac{q^{n\left(\tilde{h}_{\min }+s\right)} y^{n \tilde{h}_{\min }}}{n\left(1-q^{n}\right)\left(1-y^{n} q^{n}\right)} \\
& \approx \frac{(-1)^{2 s} \zeta(3)}{\beta(\beta+\mu)}
\end{aligned}
$$

Note that, as might have been expected, at high temperatures only the statistics of the particles matter, as $h_{\min }$ and $s$ only change the lowest states of the towers. The total free energy is now the sum over all different towers. Using table 1 we find that

$$
F \approx\left[-\left(2 h^{1,2}+2\right)+2\left(h^{1,1}-1\right)+4\right] \frac{\zeta(3)}{\beta(\beta+\mu)}=\chi \frac{\zeta(3)}{\beta(\beta+\mu)}
$$

where we used the definition of the Euler characteristic $\chi$ of the $\mathrm{CY}_{3}$. Finally we can do a Legendre transform to obtain the entropy. This is completely analogous to (2.38) and the result is

$$
S \approx(\chi \zeta(3) M(N-M))^{1 / 3}
$$

This result is equivalent to (2.38) and maximization with respect to $\mathrm{M}$ proceeds analogously, again leading to the result

$$
S(N)= \begin{cases}\left(\chi \zeta(3) \frac{N^{2}}{4}\right)^{1 / 3} & \text { if } N \leq I \\ \left(\chi \zeta(3) \frac{I}{2}\left(N-\frac{I}{2}\right)\right)^{1 / 3} & \text { if } I \leq N\end{cases}
$$

This might look somewhat unfamiliar when compared with other calculations of the elliptic genus, e.g [33, 34]. This is because those calculations were all performed in the regime $N \ll I$ where the unitarity bound on the spectrum can be ignored. It is exactly around $N \approx I$ that this bound starts to be relevant leading to a different, slower, growth of the number of states in the regime $I \ll N$. Such a behavior was also seen in the computation of the elliptic genus in [35].

Note that once more our computation above only applies for $N \lesssim I^{2}$ as the asymptotic form of the free energy is essentially the same as that of the dipole halo system.

\subsubsection{Comparison to black hole entropy and the stringy exclusion principle}

As we have seen, calculating the number of free supergravity states at fixed total charge in the large $N, M$ limit proceeds rather analogously to the counting of section 2.2.3 and, 
more importantly, we found a precise match between the leading contributions, up to an overall prefactor.

It is not hard, however, to see that even this prefactor can be made to match. In the previous subsection we focussed on the 4 dimensional degrees of freedom of the D6 $\overline{\mathrm{D} 6 \mathrm{D} 0}$ system ignoring the fact that the D0-branes bound to the D6 $\overline{\mathrm{D} 6}$ still have degrees of freedom in the internal $\mathrm{CY}_{3}$ manifold. These internal degrees of freedom can be quantized via a $0+1$ dimensional sigma model ${ }^{8}$ on the CY. The BPS states of this sigma model correspond to the cohomology of the Calabi-Yau with even degree mapping to bosonic states and odd degree to fermionic states. Thus there are exactly $\chi$ BPS states per D0 when counted with the correct sign, $(-1)^{F}$. Including this extra degeneracy in the calculation of section 2.2.3 will lead to a match with (2.51), including the prefactor.

That the two calculations provide the same amount of states is non-trivial, since earlier we restricted ourselves to counting only states realized as a D6 $\overline{\mathrm{D} 6} \mathrm{D} 0$ system, while in the second calculation we count all free supergravity states in $\mathrm{AdS}_{3} \times \mathrm{S}^{2}$ with given momentum. This suggests that indeed the leading portion of supergravity entropy is realized as D6 $\overline{\mathrm{D} 6} \mathrm{D} 0$ configurations once backreaction is included. This is a very strong result as clearly one can think of many, much more complicated, smooth multicenter configurations with the same total charge. Furthermore, we learn from these calculations that the number of such states is parametrically smaller than the number of black hole states. This seems to strongly indicate that the generic black hole state is associated to degrees of freedom beyond supergravity.

From another perspective, however, the match between the free regime and the D6 $\overline{\mathrm{D} 6} \mathrm{D} 0$ entropy is not so surprising. If we consider first a $\mathrm{D} 6 \overline{\mathrm{D} 6}$ bound state we can use a coordinate transformation from [17] to map this to global $\mathrm{AdS}_{3} \times \mathrm{S}^{2}$. Thus we can think of the D6 $\overline{\mathrm{D} 6}$ as simply generating the empty AdS background. Recalling that D0 branes lift to gravitational shock waves in 5-dimensions one might already have anticipated that counting D0's in the D6 $\overline{\mathrm{D} 6}$ background is closely related to counting free gravitons on an $\mathrm{AdS}_{3} \times \mathrm{S}^{2}$ background. What makes the result non-trivial is that interactions are apparently not terribly relevant when counting BPS states, but then again the D0's only interact very indirectly with each other. We might, therefore, wonder if more exotic configurations, such as the supereggs of [17] or the wiggling rings of [14, 26], are perhaps not captured by the free theory and hence not subject to the bound we find above. The problem with this is that we can compute not only the entropy but also the index in both regimes and they exhibit the same leading growth. If additional supergravity configurations are to generate parametrically more states this would either require very precise cancellations (so that the index is very different from the number of states) or a phase transition at weak coupling (a phase transition in $g_{s}$, not the $N=I$ transition discussed above). Even if many states would cancel in the index, one would still need to explain why they become invisible in the limit in which interactions are turned off.

It is also somewhat intriguing to see that in the "free theory" we recover the phase transition noted in the previous section only after imposing (by hand) the CFT unitarity

\footnotetext{
${ }^{8}$ In this simplistic model we neglect more complicated interactions coming from strings stretched between the D0's and the D6's in the CY.
} 
bound suggesting that the latter is taken into account by our scaling solutions. A priori this sounds somewhat mysterious as the stringy exclusion principle was argued in [8] to be inaccessible to perturbative string theory. As noted in [7] however the multicentered solutions seem to always satisfy this bound (though there is no general proof of this). In fact, the origin of the bound in this system can simply be traced back to the fact that the size of the angular momentum equals $j=I / 2-M$, which cannot be negative, and using $M=L_{0}$ this then immediately implies that the unitarity bound will be satisfied by our solutions.

In the above, we have only counted multiparticle BPS supergravitons in 5 d supergravity. It is conceivable that additional degrees of freedom could be obtained by allowing fluctuations in the Calabi-Yau as well. For example, as we discussed, D0-branes carry an extra degeneracy corresponding to the harmonic forms on the Calabi-Yau. Though this can contribute a finite multiplicative factor to the entropy, it does not change the functional form. In addition, 5d supergravity does include all massless degrees of freedom that one gets from the reduction on the Calabi-Yau, and the other massive degrees of freedom generically do not contain any BPS states.

One might also worry that multiparticle states, which in the free theory are not BPS, become BPS once interactions are included. Though this is a logical possibility, such degrees of freedom would not contribute to the index, and therefore the estimate of the index remains unaffected by this argument.

Finally, we notice that it is possible to do similar computations for $\mathrm{AdS}_{3} \times \mathrm{S}^{3}$, which leads to the result that for $N \lesssim I, S \sim N^{3 / 4}$, while for $I \ll N \ll I^{2}, S \sim I^{1 / 2} N^{1 / 4}$. It would be interesting to reproduce these results by counting solutions of $6 \mathrm{~d}$ supergravity as well.

\section{$3 \quad$ Physical properties of scaling solutions}

Although the counting above suggests that the class of D6 $\overline{\mathrm{D} 6} \mathrm{D} 0$ solutions is not sufficiently generic to characterize macroscopic five dimensional black holes the quantization has yielded several interesting surprises that warrant further discussion. Because of the structure of scaling solutions it seems that the largest number of states reside at the $J=0$ boundary of our solution space once the charge $N$ carried by the D0 particles is sufficiently large, i.e. $N>I$. Furthermore, as discussed in [2], our quantization suggests a mass gap, for asymptotically $\mathrm{AdS}_{3}$ solutions, on the order of $1 / c$ which is somewhat unexpected from the perspective of the dual MSW CFT. ${ }^{9}$ Finally, in [2] it was found that a certain region of the $\mathrm{D} 6 \overline{\mathrm{D} 6} \mathrm{D} 0$ solution space, corresponding to nearly coincident centers generating an extremely deep throat, is characterized by an extremely low phase space density implying that, upon quantization, it is not possible to support classical states localized within this region. In this section we will explore some of these physically interesting issues further.

\footnotetext{
${ }^{9}$ In some cases one can argue using dualities that the MSW CFT admits a "long string" sector, in which case $1 / c$ is the natural value for the gap, similarly to the relation between the F1-P and the D1-D5 system. For a generic MSW CFT we are not aware of any duality which maps it into a system with an obvious long string sector.
} 


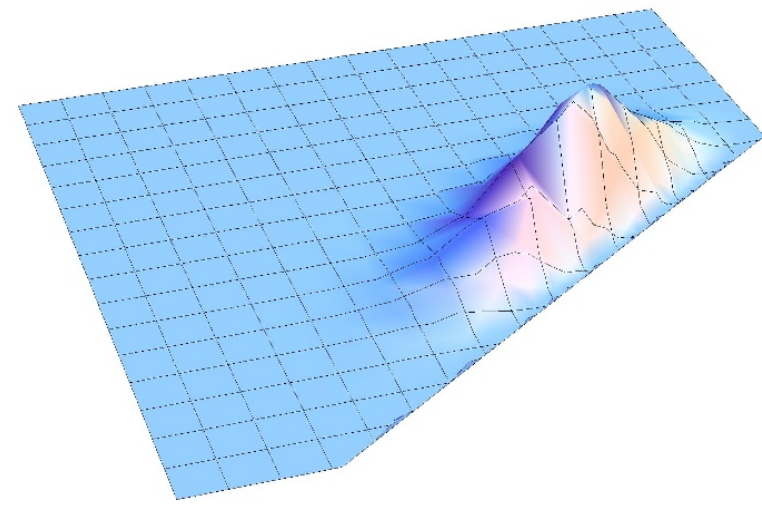

Figure 5. For a dipole halo with two D0 branes of charge $q_{1}=10, q_{2}=14$ and intersection product $I=32$ the wavefunction with quantum numbers $m_{1}=7$ and $m_{2}=6$ is plotted as a function of the coordinates $y_{1}$ and $y_{2}$. The dependence on $y$ has been removed by integration, as can be seen the wavefunction has support on a polytope of the form B of figure 4 . Note that this is one of a set of wavefunctions with zero angular momentum as $J=I / 2-3 / 2-m_{1}-m_{2}$. (A precise expression for the wavefunctions in terms of given toric coordinates can be found in [2])

\subsection{Scaling solutions with more than 3 centers}

A first generalization with respect to [2] that we considered in this paper are scaling solutions in which more than 3 centers are involved. For example in figure 4 we showed the different types of polytopes for a dipole halo with 2 D0 centers. Depending on the distribution of the D0 charge over those two centers there are now different types of polytopes with a scaling region; cases $\mathrm{B}, \mathrm{C}$ and $\mathrm{D}$ in figure 4 . As in the case of [2] with only a single D0 center, the polytope is no longer Delzant but rather rational when the scaling bound is saturated and the scaling region is an orbifold singularity. Also, similar to the case with only a single D0, the quantum wavefunctions have vanishing support on the locus classically corresponding to coinciding centers. As an example to illustrate this feature we plot such a wavefunction in figure 5 .

In this figure the region where the centers coincide classically in coordinate space, the "scaling" locus, is the diagonal edge on the right, where $x_{1}+x_{2}=I / 2$. While, this is a region corresponding to zero angular momentum in the classical theory, in the quantum theory there are intrinsic spin corrections to the angular momentum (see figure 3 and the end of section 2.1.4) so the zero angular momentum states are localized slightly away from the scaling locus. Put another way, the intrinsic spin of the centers contributes a negative amount to the total angular momentum which sets the quantum angular momentum to zero before the classical $j=0$ point (which is the scaling point) is reached. Thus, as can be checked from the form of the wavefunctions, there is zero probability to be at the classical scaling point. As in [2] we interpret this result as an indication that the classical solutions near the scaling point are highly quantum corrected, with fluctuations ranging over a large space-time volume. 


\subsection{Macroscopic quantum fluctuations from AdS/CFT}

That the quantum mechanics of scaling solutions would necessarily involve novel features was recognized shortly after their study in the AdS/CFT context as it is in this context that they present the most challenges. The existence of infinitely smooth, deep asymptotically AdS throats with low curvature everywhere seems to suggest the existence of a continuous spectrum in the dual conformal field theory. This would not agree with the fact that large black holes are dual to thermal states and at the same time carry finite entropy. It would also disagree with our knowledge of the spectrum of the D1-D5-P CFT at weak coupling.

As presaged in [22, Section 6] resolving this would require quantum effects that extend across large portions of classical, smooth solutions and this is precisely along the lines of what was found in [2] by explicit computation. The observation in [2] that the phase space volume of the system computed at weak coupling, for a system of weakly interacting D-branes, does not increase as the branes backreact and generate an infinitely deep throat follows naturally from supersymmetry but is nonetheless remarkable as it implies that the quantized "cells" of the BPS phase space stretch across macroscopic volume as an infinite throat forms. As such one might worry that this property is somehow an artifact of the BPS nature of the solutions. Here we would like to present, as supporting evidence for this phenomenon, a generic AdS/CFT based argument which uses some basic properties of the solution space, especially the fact that it is a phase space, but which does not explicitly rely on any supersymmetry.

To make an AdS/CFT based argument we of course require asymptotically AdS solutions which can be obtained by taking an appropriate decoupling limit of the solutions. Thus we assume that the total charge $\Gamma=\sum_{a} \Gamma_{a}$ has vanishing D6 charge, which allows us to take the decoupling limit of [7] and which allows us to generate a family of asymptotically $\mathrm{AdS}_{3} \times \mathrm{S}^{2}$ solutions.

The essential observation is that generic harmonics in our solutions can be expanded asymptotically as

$$
H=\sum_{a} \frac{\Gamma_{a}}{\left|\vec{x}-\vec{x}_{b}\right|}+h=\frac{\Gamma}{r}+h+\mathcal{O}\left(\frac{\left|\vec{x}_{a}\right|}{r}\right)
$$

where the terms of order zero in $x_{a}$ generate the base $\mathrm{AdS}_{3} \times \mathrm{S}^{2}$ geometry (rather they generate the geometry of an extremal BTZ black hole) and the subleading terms represent a modification of this base geometry. AdS/CFT arguments relate the expectation values of CFT operators in a particular state to subleading terms in a boundary expansion of the geometry dual to the state. In our solutions it is the terms proportional to $\left|\vec{x}_{a}\right|$ that generate these subleading terms in the expansion of the fields. For scaling solutions near the scaling point all the centers can be arbitrarily close to the origin so $\left|\vec{x}_{a}\right| \sim \lambda \ll 1$. As $\lambda \rightarrow 0$ the solution develops an infinitely deep scaling throat that closely resembles the naive black hole geometry and solutions in this region all have expectation values proportional to some positive power of $\lambda$.

Because the solution spaces we are studying map to a symplectic submanifold of the full phase space they contain both configuration and conjugate momenta variables. Hence we expect that these solutions can be parameterized, in the dual theory, by non-trivial 
expectation values of both an operator, $O$, and its conjugate momentum conjugate, $\pi_{O}$. Heisenberg's uncertainty principle, however, implies there will be an intrinsic variance in measuring these expectation values

$$
\sigma_{O} \sigma_{\pi_{O}} \geq 1
$$

The crucial observation is that this bound on the variance is finite and independent of $\lambda$ so as $\lambda \rightarrow 0$ (recall we are measuring expectation values in a state $|\lambda\rangle$ dual to a throat parameterized by $\lambda$ ) there will be some approximate value, $\lambda_{c}$, for which the variance is of the same order as either $\langle O\rangle$ or $\left\langle\pi_{O}\right\rangle$. For such states $|\lambda\rangle$ we can no longer think of the dual geometries as good classical solutions as an observer doing measurements would not be able to distinguish geometries corresponding to the different values of $\lambda$ and these look macroscopically very different.

For instance, if we consider a dipole halo with only one D0 brane then from eq. (2.4)(2.7) we see $\lambda \sim j \sim \theta_{a}$ so the depth of the throat is controlled by the distance between the D6 and the D0. This can be measured in the CFT by measuring $\hat{J}_{3}$ (referred to as $J_{0}$ in [7]). The conjugate variable in the bulk is $\sigma_{a}$ which parameterizes the phase space and also appears asymptotically in the D0 dipole moment, $\vec{d}_{0}$ (see e.g. [7, eq. (3.29)]). For scaling solutions both of these asymptotic coefficients will be first or higher order $\lambda$. Thus both $\hat{J}_{3}$ and its conjugate in the CFT will have expectation values and also variances of this order (for small enough $\lambda$ ). Even if we take the variance of $\left\langle\hat{J}_{3}\right\rangle$ to be very small in a state $|\lambda\rangle$ implying a fixed throat depth the corresponding large variance in the dual operator implies that the location of the D0 is smeared in a circle around the origin at the bottom of the throat. Recall that because of the warp factor the centers remain at a fixed, macroscopic, distance apart so the throat ends in a large quantum foam rather than a classical cap.

It would clearly be interesting to explore this argument in more general cases, to make it more quantitative and to examine its validity.

\subsection{Entropy of the $J=0$ locus}

The quantization of $\mathcal{N}=2$ solutions in [2] resolved the paradox of infinitely deep throats and the associated lack of a mass gap in the dual CFT by an explicit computation. A related question, posed in [22], is whether it is only the total angular momentum $\vec{J}$ which is quantized, or whether each of the "components" of the angular momentum, $\vec{J}_{i j}$, are separately quantized. The latter, combined with the boundedness of these angular momenta, implies that the phase space has finite volume, which in turn shows that infinitely deep throats necessarily have to cut off.

While it is clear, from AdS/CFT, if nothing else, that $\vec{J}$ should be quantized it is not clear that the $\vec{J}_{i j}$ should. From the discussion in section 2.1.4, however, it is evident that our symplectic form quantizes the angular momenta, $\vec{J}_{i j}$, between every pair of centers $i, j$, not just the total angular momenta.

To pose the problem more sharply [22] suggests that one can consider scaling solutions where the total angular momentum is zero (e.g. by imposing $J=0$ as a boundary condition) 
and then ask if the same quantization still works. That the answer will be affirmative already follows, to a large degree, from the discussion in section 2.1.4. Of course there are some subtleties that must be addressed. The $j$ term in the symplectic form, related to overall rotations of the system (this is clear in the coordinate system of section 2.1) depends on the orientation of $\vec{J}_{6 a}$. On the other hand setting $j$ to zero ${ }^{10}$ actually imposes the least restriction on the range of the $j_{a} \cos \theta_{a}$. This follows immediately from eq. (2.18) and implies that, at least in the scaling regime, a large number of states sit at the $j=0$ locus. This is can be directly seen from figure 4, e.g. case D, where the $j=0$ locus is the diagonal edge of the $y=0$ subspace (the $2 \mathrm{~d}$ polytope on the left.)

Somewhat more formally this $j=0$ submanifold is given in the polytope as the subspace $\left\{y=0, y_{1}, \ldots, y_{n}\right\}$ with $\sum_{a} y_{a}=I / 2$ and one can explicitly check that this is a symplectic submanifold of the large phase space on which the pull-back of the symplectic form is non-degenerate. For instance, in the case with two D0 centers it corresponds to the quantization of the difference $\vec{J}_{61}-\vec{J}_{62}$.

Finally, as an additional argument we quote a mathematical result of [36]. Namely, (geometric) quantization of the fixed point set of the Hamiltonian action of a compact group on a phase space yields a Hilbert space isomorphic to the invariant (under the induced group action) subspace of the Hilbert space obtained by quantizing the entire phase space.

In this case the group $\mathrm{SO}(3)$ (or its cover, $\mathrm{SU}(2)$ ), corresponding to $\mathbb{R}^{3}$ rotations, acts on the phase space and the theorem implies that rather than restrict to the $j=0$ subspace and quantize that anew we can simply use our existing quantization and extract the invariant subspace of our Hilbert space. From figure 4, cases B, C and D, it is then clear that the $j=0$ locus contributes a significant number of states.

\section{Acknowledgments}

We would like to thank I. Bena, M. Berkooz, F. Denef, E. Diaconescu, S. Guisto, J. Manschot, J. Maldacena, G. Moore and C. Ruef for interesting and clarifying discussions.

The work of JdB, SES and IM is supported financially by the Foundation of Fundamental Research on Matter (FOM). The work of DVdB is supported by the DOE under grant DE-FG02-96ER40949.

\section{A The number of states as discrete points inside the polytope}

In this appendix we show that for rational polytopes the number of normalizable modes can simply be computed from the 'discretized volume' of the polytope. This can be useful as it saves time for problems where we are only interested in the number of states and not in the explicit wave functions.

Let us very shortly review the description we use for polytopes, for the full definition and the algorithms and formulae to calculate the associated complex coordinates and wavefunctions we refer the reader to appendix B of [2].

\footnotetext{
${ }^{10}$ Note the subtlety involving the difference between the locations where the angular momentum vanishes classically and where it vanishes when intrinsic quantum spin effects are included, as explained in section 3.1.
} 
Basically, we can think of a toric polytope as a region in $\mathbb{R}^{n}$, parameterized by coordinates $x^{i}$, on which a certain set of first order polynomials are positive. That is, given such a set of $m$ first order functions:

$$
l_{j}(x)=\sum_{i=1}^{n} c_{i j} x^{i}+\lambda_{j}
$$

the polytope is defined as $P_{l}=\left\{x \in \mathbb{R}^{n} \mid l_{j}(x) \geq 0\right\}$. A few remarks are in order:

- It follows from this definition that the polytope is an intersection of $m$ half spaces.

- Note that from that interpretation it follows that $m \geq 2+n$ to have a compact polytope, so $c_{i j}$ is never a square matrix! (this has some consequences later)

- Note that $c_{i j}$ here is actually the transpose of the one defined in the appendix of [2]. This because we now use the more natural definition $c_{i j}=\frac{\partial l_{j}}{\partial x^{i}}$.

- Finally note that $c$ and $\lambda$ cannot be completely arbitrary (i.e. not every intersection of half planes gives a sensible polytope).

Example. For the readers convenience we will give the defining functions corresponding to the dipole halos of equations (2.17)-(2.18). Let us first define a coordinate system using the $n+1$ coordinates $\left(y_{0}, y_{1}, \ldots, y_{n}\right)$ corresponding to (2.16) with $y_{0}=y$.. To encode these constraints in a polytope we require an $(n+1) \times(2 n+3) c_{i j}$ matrix which which we will think of instead as $2 n+3$ vectors of length $n+1$ given below. In addition we will also need a $2 n+3$-component vector $\lambda$ with components given below as well.

$$
\begin{aligned}
\vec{c}_{0} & =(-1, \ldots,-1) & \lambda_{0} & =I / 2 \\
\vec{c}_{1} & =(1,-1, \ldots, 1) & \lambda_{1} & =I / 2 \\
\vec{c}_{2 a} & =(0, \ldots,-1, \ldots, 0) & \lambda_{2 a} & =q_{a} \\
\vec{c}_{2 a+1} & =(0, \ldots, 1, \ldots, 0) & \lambda_{2 a+1} & =0 \\
\vec{c}_{2 n+2} & =(0,-1, \ldots,-1) & \lambda_{2 n+2} & =I / 2
\end{aligned}
$$

The $\vec{c}_{2 a}$ and $\vec{c}_{2 a+1}$ are non-zero only on the $(a+1)$ 'th entry (recall that $a=1, \ldots, n$ and our coordinates are labelled from $0, \ldots, n)$. Note that the indices on $\vec{c}$ correspond to the labels $j$ in (A.1). With this in mind the reader can check that the $2 n+3$ equations defined by substituting the $\vec{c}$ and $\lambda$ above into (A.1) reproduce (2.17)-(2.18). The corresponding polytopes are shown in figure 4 .

As discussed in the appendix B of [2] and references therein, all relevant functions (i.e. complex coordinates, Kähler potential, etc.) are defined in terms of the $c$ and $\lambda$. Hence we can write the norm square of the wavefunction $\psi_{a}=\prod_{i}\left(z^{i}\right)^{\left(a^{i}\right)}$, with $a \in \mathbb{Z}^{n}$ and the $z_{i}$ appropriate complex coordinates on the toric manifold, in terms of these objects:

$$
\begin{aligned}
\left|\psi_{a}\right|^{2} \sim e^{\sum_{i} \partial_{i} g} \sqrt{\operatorname{det} \partial_{i} \partial_{j} g} e^{-\mathcal{K}} \prod_{i}\left|z^{i}\right|^{\left(2 a^{i}\right)} & \sim e^{\sum_{i} \partial_{i} g} \sqrt{\operatorname{det} \partial_{i} \partial_{j} g} \prod_{j=1}^{m} l_{j}^{\left(\sum_{i=1}^{n} c_{i j} a^{i}+\lambda_{j}\right)} \\
& \sim \prod_{j=1}^{m} l_{j}^{\left(\sum_{i=1}^{n} c_{i j}\left(a_{i}+1 / 2\right)+\lambda_{j}-1 / 2\right)}
\end{aligned}
$$


where $\sim$ indicates proportionality up to constants and functions that have no poles and also contain no overall $l_{j}$ factors. The first step is rather straightforward while the last step is more subtle to prove so that proof is relegated to a separate subsection below.

Let us first focus on the interpretation of the above result. We see that, without taking into account the fermionic contribution $e^{\sum_{i} \partial_{i} g} \sqrt{\operatorname{det} \partial_{i} \partial_{j} g}$, normalizability of the wavefunctions requires the $a \in \mathbb{Z}^{n}$ to satisfy

$$
\sum_{i=1}^{n} c_{i j} a^{i}+\lambda_{j}>-1
$$

while, when also including those necessary fermionic corrections, we find the final precise condition is

$$
\sum_{i=1}^{n} c_{i j}\left(a_{i}+1 / 2\right)+\lambda_{j}-1 / 2>-1
$$

Up to some shifts these equations essentially tell us that $a$ has to lie "inside" the polytope, making the number of states essentially the discretized volume of the polytope, i.e. the volume dived in Planck size cells. Furthermore, as we discuss in detail in the specific case studied in the main text, the shifts of $1 / 2$ introduced by taking into account the fermionic nature of the wavefunctions has a very natural physical interpretation. As was discussed in [2] the quantization of the polytopes roughly corresponds to quantizing the angular momentum of the system. That the lowest energy state corresponds to a specific alignment of the spins of the constituents then leads to various half integer shifts of the quantum angular momentum, giving rise to the $1 / 2$ 's in (A.6).

Plugging the $\vec{c}$ s and $\lambda$ 's defined in (A.2) into eq. (A.6) should allow the reader to reproduce the constraints found in $(2.20)$.

\section{A.1 Evaluation of $\operatorname{det} \partial_{i} \partial_{j} g$}

In this subsection we give a detailed description of the steps which lead from (A.3) to (A.4). These steps are based on an intermediate result, which states that

$$
\operatorname{det} \partial_{i} \partial_{j} g=\left(\prod_{j=1}^{m} \frac{1}{l_{j}}\right) A(l),
$$

where as we will show $A(l)$ is a homogeneous polynomial of order $m-n$ in the $l_{j}$ with such coefficients that for no rational polytopes it will contain an overall $l_{j}$ factor.

We will prove this in two steps. First we will evaluate the relevant determinant to show the form (A.7) explicitly. In the second step we then use this explicit form of $A(l)$ to argue its relevant properties, namely that it has no poles nor contains an overall $l_{j}$ factor.

Calculating the determinant. It is straightforward to check that

$$
\partial_{i} \partial_{j} g=\frac{1}{2} \sum_{k=1}^{m} \frac{c_{i k} c_{j k}}{l_{k}}=\frac{1}{2}\left(C \cdot L^{-1} \cdot C^{T}\right)_{i j}
$$


with $C_{i j}=c_{i j}$, remember $\operatorname{dim} C=n \times m$, and $L_{i j}=l_{j} \delta_{i j}$ an $m \times m$ matrix. So indeed $C L^{-1} C^{T}$ is a square $n \times n$ matrix and the determinant makes sense, sadly the factors inside are not square matrices making the evaluation a bit less straightforward. (We are not interested in constant factors so we will forget about the $1 / 2$ in the following)

Using the basic definition of the determinant and using some symmetry properties it is not too difficult, though maybe a bit tedious to show that

$$
\operatorname{det}\left(C L^{-1} C^{T}\right)=\left(\prod_{j=1}^{m} l_{j}^{-1}\right)\left(\sum_{S} l^{S}\left(\operatorname{det} C_{S}\right)^{2}\right) .
$$

The second factor might need some explanation as it uses some unconventional notation. The sum is over all different subsets $S \subset\{1, \ldots, m\}$ with $m-n$ elements, i.e. \#S=m-n. Furthermore we use the shorthand $l^{S}:=\prod_{j \in S} l_{j}$. Finally there is the definition of the $n \times n$ matrix $C_{S}$. Note that $C$ was an $n \times m$ matrix, $C_{S}$ is now defined as the matrix $C$ but with the $i_{1}, \ldots, i_{m-n}$ 'th columns removed where $S=\left\{i_{1}, \ldots, i_{m-n}\right\}$.

Properties of $\boldsymbol{A}(\boldsymbol{l})$. We found the result of (A.7) with the explicit form

$$
A(l)=\sum_{S} l^{S}\left(\operatorname{det} C_{S}\right)^{2} .
$$

We now want to show that $A(l)$ has no poles nor that it contains an overall $l_{j}$ factor. As is clear from its definition $A(l)$ is a homogeneous polynomial of order $m-n$ in the $l_{j}$. As the $l_{j}$ themselves are simply first order in the $x^{i}$, the polynomial $A$ has no poles in the $x^{i}$. The second point, that there is no overall $l_{j}$ factor, is more subtle to see. To show it, pick a particular element $j \in 1, \ldots, m$. By relabeling we can just take $j=1$. Now from its definition it is clear that $A(l)$ only has an overall $l_{1}$ factor if the coefficients of all the terms $l^{\tilde{S}}$, with $\tilde{S}$ such that $1 \notin \tilde{S}$, vanish.

We can now easily show that this never happens using some basic properties of $C$ and $C_{\tilde{S}}$. We will argue that there is always at least one $\tilde{S}_{\star}$ among the $\tilde{S}$ for which $\operatorname{det} C_{\tilde{S}_{\star}}$ doesn't vanish. By the definition of the $C_{S}$, all the $C_{\tilde{S}}$ include the first column of $C$, given by $c_{i 1}$. Furthermore let us go go back to the definition of $C$ and the $c_{i j}$. Note that the original definition of $c_{i j}$ was that it consisted of the $n$ components of the $\vec{c}_{j}$, which were the normals to the $m$ facets of the polytope. The statement $\exists \tilde{S}_{\star} \mid \operatorname{det} C_{\tilde{S}_{\star}} \neq 0$ thus translates to: "there exists a set of $(n-1)$ vectors among the $m$ different normals $\vec{c}_{j}$ that together with $\vec{c}_{1}$ form a basis of $\mathbb{R}^{n}$ ". We will use the notation $\vec{c}_{a}$ for these $n$ vectors and now show their existence.

Pick one of the vertices that is a corner of the facet orthogonal to $\vec{c}_{1}$ and let's call it $v_{1}$. As the polytopes of our interest are rational there are exactly $n$ edges $\vec{e}_{i}$ meeting in the vertex $v_{1}$, that furthermore form a basis of $\mathbb{R}^{n}$. Now the different facets meeting in $v_{1}$ each lie in a subspace generated by a set of $(n-1)$ of the $n$ edges $e_{i} .{ }^{11}$ So we find $n$

\footnotetext{
${ }^{11}$ Note that all facets are of this form by the definition of the edges. That furthermore each of the $n$ combinations of $n-1$ linearly independent edges generates a facet is maybe less straightforward and actually not true for a generic non-rational polytope. However here the fact that for each subspace generated by $(n-1)$ of the $n$-edges there is only one remaining edge not contained in that subset, implies that the subspace must be on the boundary of the polytope and hence generate a facet.
} 
facets that all meet in the vertex $v_{1}$. Let us label the $n$ normals to these facets as $\vec{c}_{a}$, by their definition they can be labelled such that they satisfy $\vec{e}_{i} \cdot \vec{c}_{a} \sim \delta_{i, j}$. So we see that the $\vec{c}_{a}$ form a basis of $\mathbb{R}^{n}$ that includes $\vec{c}_{1}$, which concludes the proof, i.e. we now know that $\operatorname{det} C_{\tilde{S}_{\star}} \neq 0$ for $\left(C_{\tilde{S}_{\star}}\right)_{i a}=c_{i a}$.

Open Access. This article is distributed under the terms of the Creative Commons Attribution Noncommercial License which permits any noncommercial use, distribution, and reproduction in any medium, provided the original author(s) and source are credited.

\section{References}

[1] J.M. Maldacena, A. Strominger and E. Witten, Black hole entropy in M-theory, JHEP 12 (1997) 002 [hep-th/9711053] [SPIRES].

[2] J. de Boer, S. El-Showk, I. Messamah and D. Van den Bleeken, Quantizing $N=2$ multicenter solutions, JHEP 05 (2009) 002 [arXiv:0807.4556] [SPIRES].

[3] F. Denef, On the correspondence between D-branes and stationary supergravity solutions of type-II Calabi-Yau compactifications, hep-th/0010222 [SPIRES].

[4] B. Bates and F. Denef, Exact solutions for supersymmetric stationary black hole composites, hep-th/0304094 [SPIRES].

[5] I. Bena and N.P. Warner, Bubbling supertubes and foaming black holes, Phys. Rev. D 74 (2006) 066001 [hep-th/0505166] [SPIRES].

[6] P. Berglund, E.G. Gimon and T.S. Levi, Supergravity microstates for BPS black holes and black rings, JHEP 06 (2006) 007 [hep-th/0505167] [SPIRES].

[7] J. de Boer, F. Denef, S. El-Showk, I. Messamah and D. Van den Bleeken, Black hole bound states in $A d S_{3} \times S^{2}$, JHEP 11 (2008) 050 [arXiv:0802.2257] [SPIRES].

[8] J.M. Maldacena and A. Strominger, AdS $S_{3}$ black holes and a stringy exclusion principle, JHEP 12 (1998) 005 [hep-th/9804085] [SPIRES].

[9] S.D. Mathur, The fuzzball proposal for black holes: an elementary review, Fortsch. Phys. 53 (2005) 793 [hep-th/0502050] [SPIRES].

[10] I. Bena and N.P. Warner, Black holes, black rings and their microstates, Lect. Notes Phys. 755 (2008) 1 [hep-th/0701216] [SPIRES].

[11] K. Skenderis and M. Taylor, The fuzzball proposal for black holes, Phys. Rept. 467 (2008) 117 [arXiv:0804.0552] [SPIRES].

[12] S.D. Mathur, Fuzzballs and the information paradox: a summary and conjectures, arXiv:0810.4525 [SPIRES].

[13] V. Balasubramanian, J. de Boer, S. El-Showk and I. Messamah, Black holes as effective geometries, Class. Quant. Grav. 25 (2008) 214004 [arXiv:0811.0263] [SPIRES].

[14] I. Bena, N. Bobev, C. Ruef and N.P. Warner, Entropy enhancement and black hole microstates, arXiv:0804.4487 [SPIRES].

[15] D. Gaiotto, A. Strominger and X. Yin, Superconformal black hole quantum mechanics, JHEP 11 (2005) 017 [hep-th/0412322] [SPIRES].

[16] S. Kim and J. Raeymaekers, Superconformal quantum mechanics of small black holes, JHEP 08 (2005) 082 [hep-th/0505176] [SPIRES]. 
[17] F. Denef, D. Gaiotto, A. Strominger, D. Van den Bleeken and X. Yin, Black hole deconstruction, hep-th/0703252 [SPIRES].

[18] E.G. Gimon and T.S. Levi, Black ring deconstruction, JHEP 04 (2008) 098 [arXiv:0706.3394] [SPIRES].

[19] T. Levi et al., Gödel space from wrapped M2-branes, JHEP 01 (2010) 001 [arXiv: 0909.4081] [SPIRES].

[20] I. Bena, C.-W. Wang and N.P. Warner, Mergers and typical black hole microstates, JHEP 11 (2006) 042 [hep-th/0608217] [SPIRES].

[21] F. Denef and G.W. Moore, Split states, entropy enigmas, holes and halos, hep-th/0702146 [SPIRES].

[22] I. Bena, C.-W. Wang and N.P. Warner, Plumbing the abyss: black ring microstates, JHEP 07 (2008) 019 [arXiv: 0706.3786] [SPIRES].

[23] F. Denef, Supergravity flows and D-brane stability, JHEP 08 (2000) 050 [hep-th/0005049] [SPIRES].

[24] F. Denef, Quantum quivers and Hall/hole halos, JHEP 10 (2002) 023 [hep-th/0206072] [SPIRES].

[25] J. Raeymaekers, Near-horizon microstates of the D1-D5-P black hole, JHEP 02 (2008) 006 [arXiv: 0710.4912] [SPIRES].

[26] I. Bena, N. Bobev, C. Ruef and N.P. Warner, Supertubes in bubbling backgrounds: Born-Infeld meets supergravity, JHEP 07 (2009) 106 [arXiv:0812.2942] [SPIRES].

[27] V. Balasubramanian, E.G. Gimon and T.S. Levi, Four dimensional black hole microstates: from D-branes to spacetime foam, JHEP 01 (2008) 056 [hep-th/0606118] [SPIRES].

[28] F. Larsen, The perturbation spectrum of black holes in $N=8$ supergravity, Nucl. Phys. B 536 (1998) 258 [hep-th/9805208] [SPIRES].

[29] J. de Boer, Six-dimensional supergravity on $S^{3} \times A d S_{3}$ and $2 D$ conformal field theory, Nucl. Phys. B 548 (1999) 139 [hep-th/9806104] [SPIRES].

[30] W. Lerche, C. Vafa and N.P. Warner, Chiral rings in $N=2$ superconformal theories, Nucl. Phys. B 324 (1989) 427 [SPIRES].

[31] A. Fujii, R. Kemmoku and S. Mizoguchi, $D=5$ simple supergravity on $A d S_{3} \times S^{2}$ and $N=4$ superconformal field theory, Nucl. Phys. B 574 (2000) 691 [hep-th/9811147] [SPIRES].

[32] D. Kutasov, F. Larsen and R.G. Leigh, String theory in magnetic monopole backgrounds, Nucl. Phys. B 550 (1999) 183 [hep-th/9812027] [SPIRES].

[33] D. Gaiotto, A. Strominger and X. Yin, From $A d S_{3} / C F T_{2}$ to black holes/topological strings, JHEP 09 (2007) 050 [hep-th/0602046] [SPIRES].

[34] P. Kraus, Lectures on black holes and the $A d S_{3} / C F T_{2}$ correspondence, Lect. Notes Phys. 755 (2008) 193 [hep-th/0609074] [SPIRES].

[35] J. de Boer, Large-N elliptic genus and AdS/CFT correspondence, JHEP 05 (1999) 017 [hep-th/9812240] [SPIRES].

[36] V. Guillemin and S. Sternberg, Geometric quantization and multiplicities of group representations, Inv. Math. 67 (1982) 515. 